This dissertation has been

$63-6250$

microfilmed exactly as received

JOHNSON, Garland Alton, 1936-

ISOLATION AND ANALYSIS OF MONO-, DI-, AND TRISIA LOGANGLIOSIDES.

The Ohio State University, Ph.D., 1963

Chemistry, biological

University Microfilms, Inc., Ann Arbor, Michigan 


\section{ISOLATION AND ANALYSIS OF MONO-, DI-. AND TRISIALOGANGLIOSIDES}

\section{DISSERTATION \\ Presented in Partial Fulfillment of the Requirements for the Degree Doctor of Philosophy in the Graduate School of The Ohio State University}

By

Garland Alton Johnson, B. S., M. Sc.

$* * * * *$

The Ohio State University 1963

Approved by

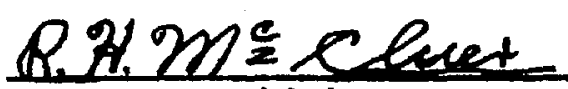
Adviser

Department of Physiological Chemistry and Pharmacology 


\section{ACKNOWLHDGRENTS}

The author wishes to express his sincere appreciation to Dr. Robert H. Micluer for the suggestion of the problem and for his guidance and encouragement throughout the course of this investigation. Thanks are also extended to Dr. K. Sambasivarao for invaluable assistance, to Dr. Keith E. IRichardson and Dr. David G. Cornwell for their many helpful suggestions, and to Dr. Ralph M. Johnson for the use of the Beckman IR-L infrared spectrophotometer.

Deepest appreciation is also extended to my wife, Ann, for the typing of this dissertation and to her and our family for the sacrifices which they have made to rnake this experience possible.

This investigation has been supported in part by a Predoctoral Research Fellowship from the Division of General Medical Sciences, National Institutes of Health. 
TABLE OF CONTENTS

Page

ACKNONLEDGMGNT . . . . . . . . . . . . . . .

LIST OF TABLES

LIST OF IIIUSTRATIONS ............ .

vi1.

STATEMFIT OF PROBLFM .................. 1

HISTORIS, ", REVIEW ................. 3

Ea: / Observations Rel: it to the Discovery of nEliosides . " . . . . . . . . . .

Natural vicurrence of Gangliosides . . . . . . . 5

Nomenclature ....................... 6

Isolation of Gangliosides ............. 7

Physical Properties of Gangliosides ... . . . . 9

Biosynthesis of Gangliosides ........... 12

Physlological Functions ............. 14

Quantitative Determinations of Gangliosides ..... 18

Iipid Components of Gangliosides . . . . . . . 19

Heterogeneity of Ganglioside Preparations ...... 21

Structure of Gangliosides ............. 26

EXPERTMENTAL ..................... 42

Analytical Methods . . . . . . . . . . . 42

N-Acetylneuraminic Acld Determination ..... 42

Hexosamine Determination ......... 42

Hexose Determination ........... 42

Glucose Determination .......... 43

Sphingosine Determination . . - . . . . 43 
TABLE OF CONTENTS (Cont'd)

Page

Phosphorus Determination .......... 43

Infrared Analysis ............ 44

Isolation of Gangliosides ............ 44

Chromatographic Procedures ............ 46

Paper Chromatographic Procedures ........ 46

Thin Layer Chromatographic Procedures ..... 46

Column Chromatographic Procedures ....... 49

Preparation of Silicte Acld for Column

Chromatography .......... 49

Preparation of Anasil s Columns ...... 49

Acid Hydrolysis Procedures in Ganglioside Analyses. . 50

Conditions for Hexosamine and Hexose Analyses. - 50

Hexosamine .............. 50

Hexose ................. 50

Determination of Hexose Ratios ........ 51

Chromatographic Frectionation of Gangliosides on

Silicic Acid Columns ............. 52

Column I............... 52

Colwain II ................ 56

Preparative Thin Layer Chromatography of Gangliosides 62

Chromatographic Fractionation of Gangliosides on

Anssil S Columns ................. 64

Column III.................... 65

Column IV ........................ 65

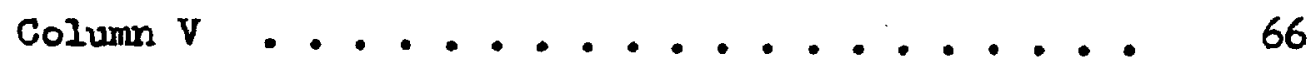


TABLE OF CONTENTS (Cont'd)

Page

Analyses of Chromatographically Homogeneous

Gangliosides ................ 67

Removal of Sillcic Acid from Gangliosides . . . 67

Characterization of 4-G.......... 69

Characterization of 3-G......... 72

Characterization of 1-G.......... 73

Characterization of FM ........... 74

Comparison of Composition for FM, 1-G, 3-G.

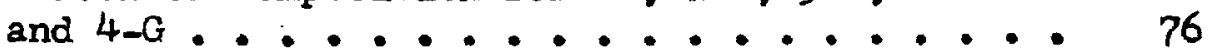

DISCUSSION . . . . . . . . . . . . . . 83

Isolation of Gangliosides ....... . . . . . 83

Chromatographic Fractionation of Gangliosides on

Silicic Acid Columns ............ 85

Preparative Thin Layer Chromatography of Gangliosides. - 86

Chromatographic Fractionation of Gangliosides on

Anasil S Colunns .............. 87

Removal of Silicic Acid from Gangliosides . . . . . 87

Analyses of Chromatographically Homogeneous

Gangliosides . . . . . . . . . . 88

Nature of Human Gangliosides . . . . . . . . 89

SUMMARY . . . . . . . . . . . . . . . . 93

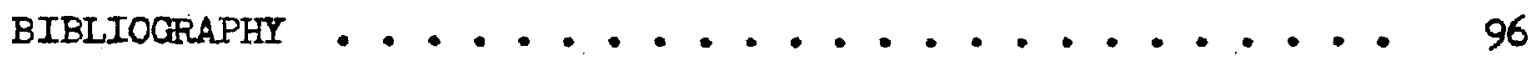

AUTOBIOGRAPHY . . . . . . . . . . . . . . 102 


\section{LIST OF TABLES}

No.

Page

1 Carbohydrate Composition of Gangliosides . . . . . . 27

2 Oligosaccharides from Acetolysis (Kuhn et al.) . . . 31

3 Composition of Bovine Gangliosides ........... 33

4 Ollgosaccharides Released from Gangllosides

During Acid Hydrolysis . . . . . ... . . 38

5 Carbohydrate Derivatives Identified from Ganglioside

Methylation Experiments ......... 40

6 Analysis of Ganglioside FM ............. 64

7 Carbohydrate Composition of Lyophilization Residue Containing 4-G.................. 69

8 Carbohydrate Composition of 4-G .......... 72

9 Carbohydrate Composition of 3-G .......... 73

10 Carbohydrate Composition of 1-G ........... 74

11 Initial Carbohydrate Composition of FM . . . . . . 74

12 Carbohydrate Composition of FM ........... 75

13 Carbohydrate Composition of Four Human Brain

Gangliosides.............. 77

14 Comparison of Gangliosides Reported by Various

Investigators ................... 92 


\section{LIST OF IILOSTRATIONS}

No.

Page

1 The Sialic Acid, N-Acetylnouraminic Acid ......... 5

2 Ganglioside by Klenk and Lauenstein .......... 26

3 Repeating Unit of Ganglioside (Bogoch) . . . . . . . 28

4 Structures for Gangliosides (Klenk and Geilin) ...... 30

5 Ganglioside $G_{2}$ (Kuhn et 2l.) . . .......... 32

6 Asialoganglioside of Svennerholm .......... 36

7 Preparation of Gangliosides from Normal Adult

Human Brain Tissue ............. 47

8 Ganglioside Elution from Column I . . . . . . . . 53

9 Ganglioside Elution from Column II . . . . . ... 58

10 Thin Layer Chromatography of Fractions from Column II . . 60

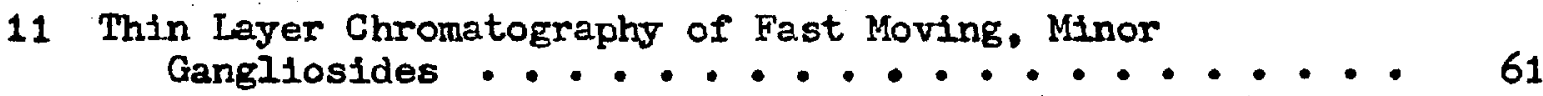

12 Thin Layer Chromatography of Homogeneous Gangliosides (I). 70

13 Thin Layer Chromatography of Homogeneous Gangliosides (II) 71

14 Infrared Spectrum of Ganglioside FM . . . . . . . . . 78

15 Infrared Spectrum of Ganglioside 1-G. . . . . . . 79

16 Infrared Spectrum of Ganglioside 3-G . . . . . . . 80

17 Infrared Spectrum of Lyophilization Residue

Containing Ganglioside 4-G .......... 81

18 Infrared Spectrum of $\mathrm{N}$-Acetylneuraminic Acid . . . . 82 


\section{STATEMENT OF PROBLEM}

Ganglioside was first described by Klenk (1) in 1935 and by: Blix (2) in 1938. Klenk (3) then isolated large amounts of ganglioside from the bratn of a Tay-Sachs' victim. This glycolipid was characterized by its content of sialic acid, an acidic carbohydrate which was responsible for the characteristic orcinol color reaction.

During the years that followed few reports concerning ganglioside were noted. In this interim ganglioside was thought to be a single glycolipid containing sphingosine, stearic acid, galactose, glucose, galactosamine, and sialic acid. The molecular weight of this glycolipid as determined by sedimentation and ultracentrifugation in aqueous media ranged from 100,000 to 800,000 . From these data ganglioside was postulated to be polymeric in nature.

Shortly before this investigation began, the molecular weight of ganglioside was determined by ultracentrifugation in an organic solvent to be approximately 1500. The heterogeneity of ganglioside was also reported with the number of components-ranging from two to eight. A variety of methods were introduced for the isolation of gangliosides, but the composition reported by various investigators was not in agreement.

This investigation wds designed to confirm or disprove some of the reports which were in conflict regarding the number of components 
and their composition. It was first necessary to devise a method for the fractionation of gangliosides into individual chromatographically homogeneous components in sufficient quantities to permit definitive characterization. 


\section{HISTORICAI REVIEN}

Early Observations Related to the Discovery of Gangliosides In $1927 \mathrm{Walz}$ (4) attempted to isolate the cerebroside kerasin from cow's milk and succeeded in obtaining a very small yield of a substance which gave a red-violet color with Bial's reagent (orcinol and ferric chloride in hydrochloric acid) (5) in contrast to the green color which results in a similar reaction with cerebrosides. Wali also reported finding a substance with similar characteristics in a brain extract which produced heavy black flocks upon hydrolysis with sulfuric acid. In the same year Levene and Landsteiner (6), noting the report by Walz, described the isolation of a new water soluble lipid from horse kidney. This lipid also produced a purplish red coloration upon heating with Eial's reagent. The elementary composition differed from that obtained from cerebroside and from sphingomyelin. The products of hydrolysis, sphingosine, fatty acids and a sugar, most likely galactose, were identical to those obtained by Walz (4).

In 1935 klenk (I) isolated from the brain of a Niemann-Pick disease victim a lipld with similar properties. Soon thereafter Klenk $(7,8)$ was able to show that this lipid was present in increased amounts in the brain of a Tay-Sachs' disease (infantile amaurotic 1diocy) victim. This new lipid, which he labeled 
"substance X", made up the major portion of the protagon fraction. 1 Later Klenk and Langerbeins (9) presented evidence that indicated "substance $X "$ was localized in the ganglion cells in amounts which caused these cells to appear distended. Due to the localization of this substance in the ganglion cells, Klenk (3) gave to this new water soluble Iipid the name "ganglioside". Upon acid hydrolysis the ganglioside preparation yielded stearic acid. sphingosine, galactose, glucose, and neuraminic acid ${ }^{2}$, the sugar moiety which was responsible for the characteristic color reaction with orcinol. This new sugar was isolated as the methoxy derivative $(10)$.

During this same period Blix (2) reported the isolation of a ganglioside preparation from normal brain tissue; he concluded that ganglioside contained hexosamine, in addition to the hydrolysis products noted by Klenk, as a characteristic component. The hexosamine from ganglioside hydrolysates was characterized as galactosamine $(11,12)$.

$1_{\text {The term protagon fraction as used by Thierfelder (13) referred }}$ to that portion of the acetone insoluble and ether insoluble residue of cerebral tissue which was extracted with hot ethanol. This fraction consisted essentially of the sphingolipids, namely. cerebrosides, gangliosides, and sphingomyelins.

${ }^{2}$ Since the initial report concerning the isolation of neuraminic acid (10), a group of substituted neuraminic acids have been shown to exist in nature. The name neuraminic acid is now reserved for the basic, unsubstituted structure which is common to all the derivatives. The various $\mathrm{N}$-acylated and $\mathrm{O}$-substituted derivatives of neuraminic acid, collectively termed slalic acids, are designated according to the nature of the Iinkage, 1.e.. N-acetylneuraminic acid, N,O-diacetylneuraminic acid. The chemistry, natural occurrence, and functions of the sialic acids have been recently reviewed (14). 
Natural Occurrence of Gangliosides

Since the initial isolation of this water soluble sphingolipid from human brain, similar sphingolipids containing sialic acid have been isolated from bovine spleen (15), human spinal cord (16), and from equine $(15,17)$, bovine (18), and canine (19) erythrocyte stroma.

While gangliosides have not been reported in cow's milk, several sialic acid containing oligosaccharides have been isolated from both cow's milk and human milk (20) which may explain the effect noted by WaIz (4).<smiles>CC(=O)NC(O)C(O)C(O)C(O)C(O)C(=O)C(=O)O</smiles>

Figure 1.--The sialic acid, N-acetylneuraminic acid (NAMA), according to Kuhn and Erossmer (21)

In 1951 a high molecular weight substance resembling ganglioside in composition was isolated from human gray matter by Folch et al. (22) and called strandin. It was reported that this water soluble and also chloroform/methanol soluble strandin contained only a very small amount of sialic acid (1-2 per cent) in addition to fatty acids; a sphingosine-like substance and other carbohydrate components. However, the very low sialic acid content in the strandin preparations was later demonstrated to be inaccurate $(23,24)$. Subsequent work indicates strandin and ganglioside to be indistinguishable $(25,26)$. 
The term strandin, while inferring individuality, is currently employed synongmously with gangliosides (27).

Rosenberg and Chargaff $(28,29)$ isolated a mucolipid from ox brain which, when purifled, was reported to contain approximately 5 per cent amino acids in addition to sphingosine, fatty acids, * galactosamine, and sialic acid. Their difficulty in freeing this substance of amino acids led the authors to believe that this mucolipid represented a new entity. However, the amino acids present in their preparation have not been shown to be covalently bound to the glycolipid. Whether the mucollpid is a glycolipid, or ganglioside, contaminated with proteinaceous material, or whether the mucolipid is a true entity with covalently bound amino acids or peptides is a problem that remains to be solved.

\section{Nomenclature}

The term ganglioside has been employed in referring to a group of glycosphingolipids which contain sialic acid irrespective of their source; this usage corresponds to that used by Klenk and Debuch (31). All of the compounds which have been mentioned above can be included in this classification and are distinguishable from other glycosphingolipids which do not contain sialic acid. This latter group includes the human blood group (A-positive and B-positive) substances (31), "globosides" from bovine erythrocytes (32), whe polycerebrosides (33), and the asialogangliosides isolated from Tay-Sachs' brains (34). Henceforth in order to provide a more definitive meaning, the term gangliosides in this thesis will refer 
to a group of closely related glycosphingolipids containing sialic acid and which are native to or are isolated from the central nervous system.

\section{Isolation of Gangliosides}

The methods employed for the isolation and purification of gangliosides have been quite varied. Consequently, some confusion in interpretation has resulted in the descriptions of the products which have been obtained.

Initially Klenk (3) extracted principally the protagon fraction (see footnote $\mathrm{p} .4$ ) with chloroform/methanol. His isolation of gangliosides from this fraction was based upon their solubility as lead salts in methanol/benzene, 1:1, and their insolubility in 95 per cent acetone which removed contaminating cerebrosides. A separation of gangliosides from phosphorus-containing impurities was achieved by chromatography on an alumina oxide column and elution of the gangliosides from the column with pyridine.

Probably the most widely employed method for the preparation and purification of brain lipids including gangliosides is that introduced by Folch et al. $(22,35)$. This method consists of homogenizing the tissue with a 20 fold volume of chloroform/methanol. $2: 1(v / v)$, and washing the extract by the addition of either water or an appropriate salt solution ( 0.2 of the volume). A two phase system results in which the non-lipid contaminants are in the upper phase and the lipids are quantitatively partitioned into the lower phase. However, because of the hydrophilic nature of the carbohydrate groups present in the molecule, gangliosides partition into 
the upper aqueous phase. The addition of $\mathrm{NaCl}$ or $\mathrm{KCl}$ to the aqueous wash (approximately 0.1 per cent solutions) apparently facilitates the partitioning of the gangliosides into the aqueous layer. The solvent ratios in this two phase system can also be produced by mixing chloroform/methanol/water, $8: 4: 3(v / v / v)$, and used for partitioning lipid extracts.

The isolation of the ox brain mucolipid by Rosenberg and Chargaff (29) employed a chloroform/methanol, 2:1, extraction of wet tissue followed by dialysis partitioning of the extract between the aqueous and the chloroform layers. This mild extraction and isolation procedure was comparable to that of Folch et al. (35).

Bogoch (26) employed a hot methanol extraction of whole ox brain cerebral hemispheres followed by a precipitation of the extracted material in the cold and subsequent partitioning of the residue between the two phases of the Folch solvent (35). The upper phase material, freed from solvents and dialyzed, was referred to as crude brain ganglioside. This crude material was further purified by repeeted partitioning between the two phases.

Svennerholm (36) described a procedure in which humen gray matter was homogenized and dried with acetone. This dried residue was extracted with chloroform/methanol, 1:2, in a soxhlet extraction bulb. After evaporation of the solvent the lipid residue was dissolved in a chloroform/ethanol/water solvent and subjected to chromatography on a cellulose column; two ganglioside fractions were obteined in the eluted material.

Experiencing some difficulty in isolating gangliosides from brain slices or homogenates by the methods of Klenk and Langerbeins (9) 
or Folch et al. (22). Trams and Iauter (37) described recently a new method for ganglioside isolation in preliminary biosynthetic investigations. Brain homogenates were extracted with aqueous tetrahydrofuran, the extract was washed with ether and then deionized on a mixed-bed ion exchange column. The barium salts of gangliosides were precipitated in the cold by the addition of barium hydroxide and cold ethanol. The barium was removed as the sulfate by centrifugation. The yield of gangliosides by this method compared quite favorably with other methods $(20,29,35)$. The large volumes required in this method make prohibitive its application to anything but small tissue samples.

Other investigators have applied various chromatographic teohniques for the separation of gangliosides from other brain lipids. Payne and Platt (38) used silicic acid column chromatography to resolve crude sphingolipid extracts from dog and rat cerebral tissues into two ganglioside fractions in addition to several other fractions. Cellulose column chromatography has also been used by Rouser et al. (39) to separate gangliosides from other cerebral lipids.

\section{Physical Properties of Gangliosides}

The physical properties of ganglioside preparations have also been a source of controversy among investigators working in this area of biochemistry. The molecular weight determinations of gangliosides by ultracentrifugation in aqueous solutions have ranged from 250,000 for strandin (22) and the ganglioside preparation of Bogoch (26) to somewhere between 100,000 and 800,000 (40). 
The large molecular weight led many investigators to theorize that gangliosides existed as high molecular welght polymers. On the basis of molecular weight determinations and hydrolytic data Bogoch (26) postulated a probable repeating unit with a molecular weight of 3314 for a macromolecular polymer of ox brain ganglioside. Substituting $N, N^{\prime}$-dimethylformamide for water as the solvent for ultracentrifugal studies of a ganglioside preparation, Klenk and Geilin (40) found a molecular weight in the range 1000-2000. An equivalent weight of 1226 to 1510 was determined from titration data. Egge (48) employed simflar conditions in obtaining a molecular weight of 4000-5000 and an equivalent weight of 1390-1410 for his ganglioside $G_{2}$ (since relabeled $G_{1}(41)$ ). Klenk (40) proposed that gangliosides existed in aqueous solutions as large aggregates, or associated micelles, which explained the large "molecular weights". Hydrophoblc forces (42) acting on the lipid moiety are envisaged as being responsible for the formation of these aggregates. Thus organic solvents promote the formation of monomolecular units, and true molecular weights are obtained. The fact that no sedimentation of gangliosides was noted at a methanol. concentration of fifty per cent in N,N-dimethylformamide and in tetrahydrofuran substantiate this contention (37). This micellar concept has now been generally accepted. The axial ratio of the ganglioside micelle in water has been estimated to be about 1.2 (37). Therefore homogeneity of ganglioside preparations by electrophoresis and ultracentrifugation in aqueous solvents are not valid criterla of purity. 
The interaction of gangliosides with various cationic molecules has also been investigated. The micellar formation of gangliosides in aqueous solvents with the sialic acid residue providing an anionte charge on the periphery of the micelle is an important concept in these relationships. Harris and Salfer (47) studied the interaction of gangliosides with a group of cationic dyes. This interaction resulted in a shift of the absorption spectrum of the dye in the direction of shorter wave lengths. The metachromatic reaction increased as a function of ganglioside concentration. However, very high concentrations decreased this metachromatic effect. The basic proteins, protamine base, protamine sulfate, and lysozyme, showed marked interaction with gangliosides as evidenced by turbidimetric measurements (43). The fluorescence quenching effects of gangliosides with certain acridine dyes was also investigated (44).

Similarly the formation of ganglioside-cationic dye complexes has been utilized by Albers and Koval (45) to determine the relative binding affinities of other cations with gangliosides. The fact that gangliosides can form complexes with other naturally occurring lipids (lecithin and cerebrosides) indicates that both the lipid moiety and the anionic charge on the carboxyl group of the sialic acid residue are important in determining its complexing properties.

The differential solubility of gangliosides from normal brain tissue and abnorral gangliosides of Tay-Sachs' diseese victims has been described $(34,46)$. Gatt reported that whereas normal gangliosides were readily distributed into the aqueous phase of 
the saline containing Folch solvents (35), the abnormal Tay-Sachs' ganglioside remained in the chloroform-rich layer. However, if water was used instead of the saline solution in establishing the two phase system, the abnormal ganglioside was distributed into the aqueous layer. Chloroform soluble complexes of gangliosides were formed by the addition of purified lipids (cerebrosides, cephalins, sphingomyelins, and lecithins) to saline-methanolic solutions of gangliosides. These data point to the formation of natural ganglioside, lipid, and salt complexes.

\section{Biosynthesis of Gangliosides}

Studies related to the biosynthesis of gangliosides have been undertaken, especially in regard to the source of the carbohydrate portion of these glycolipids. Uniformly labeled glucose-c14 and galactose-c ${ }^{14}$ were incorporated into rat brain gangliosides after intracerebral or intraperitoneal administration. The incorporation. which was most rapid in six to ten day old animals, was found principally in the galactose and galactosamine residues. Mandelstam and Burton (49) detected very little incorporation in the glucose or sialic acid residues from either of the sources. In mice sacrificed one hour after intraperitoneal administration of labeled glucose or galactose, Moser and Karnovsky (50) found that gangliosides were more effectively labeled from galactose. Glucose-C $\mathrm{C}^{14}$ similarly administered was more readily incorporated as a unit into mice cerebrosicles than was galactose. 
The steps in the biosyñthesis of cerebrosides have been summarized by Eredy (51) as follows:

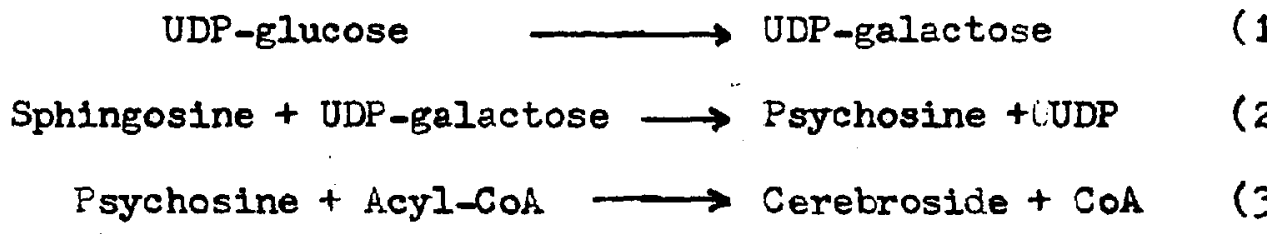

Because of the presumed similarity in the lipophilic portion of the ganglioside molecule and the cerebrosides, the role of cerebrosides as biosynthetic precursors of gangliosides has been suggested. The fatty acid composition of rat brain cerebrosides which was reported by Kishimoto and Radin (52) indicated a predominance of saturated $\alpha$-hydroxy acids, principally $c_{22}$ and $c_{24^{*}}$ Saturated normal acids (mainly $\mathrm{C}_{24}$ and $\mathrm{C}_{<20}$ ), unsaturated normal acids, and unsaturated $\alpha$-hydroxy acids (principally $\mathrm{C}_{24}$ and $\mathrm{C}_{22}$ ) were detected in lesser amounts. The investigation of the fatty acid composition in cerebrosicies was extended to human brain cerebrosides by Radin and Akahor1 (53). The $\alpha$-hydroxy acids retained their predominance, and the principal saturated acids were stearic and Iignoceric $\left(C_{24}\right)$. The heterogeneous nature of the ceramide portion of the cerebrosides contrasts the very high stearyl-sphingosine content of the gangliosides. The presence of stearic acid in the brain cerebrosides is not sufficient evidence to either negate or implicate cerebrosides as biosynthetic precursors of gangliosides.

The presence of galactocerebrosfdes in ganglioside hydrolysates remains in doubt $(40,54)$. The structural formulae which have been proposed for gangliosides a.ll feature a glucose unit linked 
glycosidically to the prinary hydroxyl group of sphingosine. Thus psychosine does not appear to be involved to any major extent in the biosynthesis of gangliosides.

The increasing speculation of potential involvement of gangliosides in physiological processes together with improved and standardized isolation techniques should soon provide a detailed sequence of ganglioside biosynthesis.

\section{Physiological Functions}

Knowledge concerning the physiological functions of gangliosides is still quite speculative.- Their postulated activity is usually related to the sialic acid residue which is antonically charged at physiological pH. However, the ability of gangliosides to be accommodated into lipid complexes in addition to their tendency to combine reversibly with many large organic cations led Albers and Koval (45) to suggest the possible participation of gangliosides in biological membranes.

Cerebral tissues incubated in oxygenated glucose media normally respond to electrical impulses by a number of metabolic changes, including an elevation of their sodium ion content and a simultaneous decrease in their potassium content (55). This response of the tissue to electrical impulses was inhibited by incubation of the tissue with added basic proteins or by maintaining the tissue at $00 \mathrm{C}(56)$. Excitability can be restored to these tissues by incubation with various sialic acid containing glycolipids or glycoproteins. Gangliosides were found to be the most effective of the compounds investigated for their ability to restore the 
metabolic response to electrical impulses (57). Wolfe and McIlwain (58) observed that native basic proteins were lost from nuclei and appeared in the microsomal fraction in tissue which had been kept in the cold, then homogenized and subjected to differential ultracentrifugation. The reentry of potassium ions back into the cerebral tissues after stimulation was resumably blocked by this migration of basic proteins. These results suggested a movement of basic proteins away from the nuclel during excitation and subsequent combination with acidic substances, possibly native gángliosides, present in the lipid-rich membrane constituents of the cytoplasm (58). This postulated complexing role was strengthened with information concerning the intracellular distribution of gangliosides. Wolfe (59) demonstrated by differential ultracentrifugation of of guinea pig cerebral cortex homogenates that the gangliosides existed principally in the microsomal and post-microsomal fractions rich in membranous components. This microsomal fraction was the most effective subcellular fraction in removing from solution added cerebral cortex histones ( 58$)$.

Additional evidence of intracellular ganglioside-basic protein complexes has been reported which may implicate this phenomenon in active cation transport. The addition of basic protamines to brain tissue substantially reduced the extractablifty of gangliosides by chloroform/methanol, $2: 1(56,60)$. A similar addition of protamine to a suspension of brain subcellular particles completely prevented the extraction of gangliosides (61). Gangliosides which were added to a brain homogenate were wholly recovered from the nuclear fraction (6I). 
Järnefeldt (62) demonstrated that microsomal fractions of cerebral tissue homogenates contain an adenosine triphosphatase (ATPase) which is activated by sodium 1ons. McIlwain's results (55-58) suggest that both the basic proteins and gangliosides modify the cation transport in which this microsomal ATPase is postulated to be involved. Deul and MeIlwain (63) observed that the sodium activated ATPase of rat brain microsomes was inhibitéd by gangliosides. Protamine exhibited no effect upon the enzymic activity. Emphasizing the possible function of gangliosides in the process of active cation transport. Hiliman (64) found that the membrane potentials of guinea pig cerebral cortex preparations were affected by gangliosides and basic proteins. The cerebral cell membranes which were hypopolarized by clupein sulfate, a basic histone preparation from herring sperm, were repolarized by the addition of gangliosides. These results parallel the finding noted above that gangliosides restored the metabolic response to electrical impulses of histone inhibited cerebral tissues (57). This report by Hillman (64) further supported an assertation of Thomson and McIlwain (65) that gangliosides and protamines act upon the cell membrane in the cation movement accompanying tissue excitability.

The interaction of gangliosides and numerous cationic dyes and other organic cations has already been mentioned in the discussion of the physical properties of gangliosides.

Several sialic acid containing mucoprotelns competitively inhibit the hemagglutinating ability of heat treated influenza virus. This inhibitory activity is lost upon treatment of the 
mucoprotein receptors with neuraminidase. However, this inhibition of viral hemagglutination is not a characteristic of only sialic acid containing mucoprotelns. The mucolipid of Rosenberg and Chargaff (29) inhibited viral hemagglutination (24). This property of the mucolipid was lost by removal of a large percentage of the sialic acid by viral neuraminidase. A ganglioside preparation of Bogoch exhibited slight inhibition of viral hemagglutination (66). No inhibition of viral hemagglutination was demonstrated with a ganglioside preparation from a Tay-Sachs' brain (24).

The specific fixation of tetanus toxin by ox gangliosides was reported by van Heyningen and Miller (67). North and Doery (68) reported that ganglioside preparations incubated at $37^{\circ}$ with staphylococcal, tetanus, and diphtheria toxin inactivate the toxins as evidenced by tests in experimental animals. Van Heyningen (69) demonstrated by ultracentrifugation that bovine brain gangliosides, which fixed tetanus toxin, did not fix diphtheria toxin. The capacity of gangliosides to fix tetanus toxin was related to the sialic acid content (70). Inactivation of diphtheria toxin by ganglioside as observed by North and Doery was, according to van Heyningen, nonspecific and independent of fixation (69). The presence of a neuraminidase in diphtheria toxin (71) may explain the failure of gangliosides to fix diphtherla toxin. Burton and Balfour (72) recently observed no direct relationship between the lethal action of tetanus toxin and the total ganglioside content in young rat brains: During the period of inrestigation a sixfold increase in ganglioside content and a twofold increase in body welght were 
noted, whereas no change in minimal lethal dose of tetanus toxin was found. This latest report suggests that the toxin complexing substance may not be solely gangliosides.

Bovine brain gangliosides have been shown to reduce the neuromuscular blocking activity of d-tubocurarine (curare) and certain other diquaternary aromatic nouromuscular blocking agents (73). Because of the lack of influence of gangliosides upon the activity of acetylcholine or carbemylcholine which presumably act at the same tissue sites as the blocking compounds, the observed action of gangliosides was most likely an interaction between the neuromuscular blocking agents and the gangliosides.

The relation of gangliosides to the $R h_{0}(D)$ antigen has been very briefly described by Johnson and MoCluer (74). Crude preparations of bovine gangliosides weakly inhibited the agglutination of $\mathrm{Rh}_{0}(\mathrm{D})$ antigen by $\mathrm{Rh} / \mathrm{D}$ ) antibody. Yokoyama and Trams (116) have noted similarities in the end group configuration of calf brain gangliosides and $R h_{0}(D)$ antigen.

Quantitative Determinations of Gangliosides

Because of the distinguishing and peculiar presence of sialtc acid in gangliosides, most of the quantitative determinations of gangliosides have been based upon their content of siallc acid. The Initial estimation of gangliosides described by Klenk and Langerbeins (9) was based upon the determination of sialic acid by the orcinol reaction in the ether and acetons insoluble residue from a chloroform/methanol extract. Svennerholm (75), noting that 
the ether extractions in the above method resulted in a sizeable loss of gangliosides, described a procedure which eliminated the ether extractions and employed a modified orcinol neaction. Interferring unsaturated fatty acids were eliminated by selective hydrolysis and petroleum ether extraction. Long and Staples (76) based a quantitative determination of gangliosides upon the sialic acid which was partitioned into the upper phase of the Folch solvents.

Improved methods for the quantitative analysis of sialic acid have onhanced the accuracy and reproduclbllity of these determinations. Among these improved procedures are the resorcinol assay of Svennerholm (77) with its modification by Miettinen and TakkiIuukkainen (78), and the thiobarbituric acid (TBA) assay of Warren (79), which measures only free sialic actd and thus requires preliminary hydrolysis of the gangliosides.

Itpid hexosamine determinations have also been used for calculating ganglioside content in cerebral tissues (80). However, this application for the quantitation of gangliosides does not comply with the definition which has been designated.

A recent report by McCluer et 21. (81) has described the adsorption of gangliosides upon sillcic acid for the specific determination of ganglioside-bound sialic acid.

\section{Lipld Components of Gangliosides}

Blenk (3) reported only the presence of stearic acid as the fatty acid molety in human ganglioside hydrolysates. Greater 
heterogeneity in the fatty acids of human gangliosides was demonstrated by gas-liquid chromatography (OLC) by Klenk and Geilin (54). The values obtained were 1 per cent palmitic, 94 per cent stearic, 4 per cent arachidic, and 1 per cent behenic $\left(C_{22}\right)$. Trams et al. (82) have analyzed the fatty acids of gangliosides from rartous species by GLC. The variation in the percentage composition of fatty acids was less than might be anticipated with the wide range of species selected for Investigation. Stearic acid was the major fatty acid in each species. The other principal acids identified for all species studied were palmitic, arachidic, and behenic, except for the shark in which case a $C_{24}$-monounsaturated fatty acid made up 13 per cent of the total fatty acid composition. The fatty acid composition of human gangliosides was 86 per cent stearic, 10 per cent arachidic, and 3 per cent behenic. The long chain base present in gangliostdes had originally been assumed to be only sphingosine. Becently the presence of minor amounts of dihydrosphingosine in ganglioside preparations from various species was noted (82). Klenk and Geilin (83) reported that upon ozonolysis of the sphingosine molety of bovine gangliosides small amounts of palmitic acid in addition to myristic acid were formed. This cleavage of the long chain base at the double bond to produce palmitic acid inferred that a $C_{20}$ homologue of sphingosine was present in bovine gangliosides. This corresponded to the earliex observation of Prostenlk et al. (84) who found such a sphingosirie base in sphingolipld fractions of bovine and equine 
brains. However, similar treatment of the sphingosine preparation of human gangliosides was reported to produce only myristic acid (83) which would indicate the presence of only $\mathrm{C}_{18}$ sphingosine.

Although the similarity between the bovine brain mucolipid (29) and gangliosides has not been assured and is not assumed, it is significant that Stanacev and Rosenberg (85) have reported the presence of a $\mathrm{C}_{20}$ homologue of sphingosine in their mucolipid preparation; this homologue was called icosisphingosine. Perlodate oxidation of the sphingosine preparation and subsequent gas chromatographic analysis of the resulting aldehydes produced the following data for the long chain base composition.

$\begin{array}{lr}\text { Dihydrosphingosine } & 3.5 \text { per cent } \\ \text { Sphingosine } & 50.0 \text { per cent } \\ \text { Icosisphingosine } & 46.5 \text { per cent }\end{array}$

Heterogeneity of Ganglioside Preparations

Brain gangliosides were long considered to contain a uniform carbohydrate molety (86). More than two decades have now passed since the initial detection of gangliosides by Klenk (1) and by BIIx (2). Although the concept of the composition of gangliosides has been altered little during this time, the heterogeneity of the preparations has been assured. The number of gangliosides resolvable by the various fractionation techniques and their composition has been and continues to be the focal point for many current investigative endeavors. The problem 1s, no doubt, made more complex and confusing due to the variation in the methodology of 1solation, and the efficacy of resolving ganglioside preparations into their individual compounds. 
Svennerholm (36) was the first to demonstrate the heterogeneous nature of a ganglioside preparation. Two normal human brain ganglioside fractions which differed in thetr hexosamine:sialic acid ratios were eluted from a cellulose column. Shortly after this Meltzer (87) reported the presence of at least elght components in a strandin preparation subjected to a three phase solvent distribum. tion. Further descriptions of these components have not yet been reported.

Kuhn (88) reported the separation of beef brain gangliosides into two components by paper chromatography. The two fractions were designated $G_{2.0}$ and $G_{0.5}$ due to their chromatographic mobilities as compared to a lactose standard. These two components were later relabeled $G_{2}$ and $G_{1}$, respectively $(20)$. An added note to this report briefly mentioned the separation of the ganglioside $G_{1}$ by cellulose column chromatography into three homogeneous components. Kuhn et al. (4l) then recrystallized four gangliosides and extended their means of resolution to paper chromatography and thin layer sillcic acid chromatography. These authors also noted that human brain gangliosides were chromatographically identical to the bovine gangliosides. The method employed for the extraction and isolation of gangliosides by these investigators has not yet been published. During this same period a human ganglioside preparation of Klenk and Geilin (40) was subjected to selective degradative acid hydrolysis, periodate oxidation, and methylation followed by hydrolysis. On the basis of their data two formulae were proposed for the constituent gangliosides of their preparation. One of the 
compounds was stated to contain galactosamine wh1le the other lacked galactosamine. More recently an acetylated ganglioside preparation was separated into two fractions by chromatography on a cation exchange resin column (89). The first fraction (Fraction II) contained es.sentially hexosamine-free ganglioside material which was further resolved into two distinguishable components by paper chromatography. The second fraction (Fraction III) contained hexosamine and was also shown by paper chromatography to consist of two components. Klenk and Geilin (54) have now fractionated a human ganglioside preparation into four gangliosides by silicic acid column chromatography. The two fastest moving gangliosides (A and B) contained hexosamine, whereas the two slowest (C and D) did not. However, only two (A and D) were chromatographically homogeneous. Utilizing column chromatographic techniques, Svennerholm and Raal (90) fractionated on silicic acid a human ganglioside preparation into two principal fractions, a fast moving and a slow moving with reference to their chromatographic mobilities. Similar results were obtained from calf brain gangliosides subjected to the same treatment. Because of the sialic acid:sphingosine ratio of 1 in the fast moving and approximately 2 in the slower moving, the fast moving was designated monosialoganglioside and the slower as disialogangliom side. Both fractions were shown to be heterogeneous by paper chromatography. The slower moving gangllosides were converted to monosialogangliosides by weak acid hydrolysis and by neuraminidase treatment.. The Tay-Sachs' ganglioside which had the fastest mobility of the gangliosides isolated was assumed to be a monosialoganglioside. 
Van Heyningen and Miller (68) prepared bovine gangliosides employing a combination of earlier described procedures $(22,29,36)$. Paper chromatography of this preparation showed both fast- and slow-moving material when sprayed with a cresyl violet spray reagent; the "fast" gangliosides consisted principally of non-metachromatic material and the "slow" mainly of metachromatic material. Both components had equal hexosamine content, but the "slow" material contained more sialic acid ( $s e$ Table 1) and possessed a greater tetanus toxin fixing ability. When compared paper chromatographically with the mucolipid of Rosenberg and Chargaff (29) and the total ganglioside preparation of Klenk and Geilin (40), the mucolipid was mainly "slow" and metachromatic, whereas Klenk's preparation was principally "fast", non-metachromatic, and contained less slalic acid than either the "fast" material of van Heyningen (67) or the mucolipid. A sketch representing the paper chromatographic resolution of the van Heyningen gangliosides exhlbited five cresyl violet staining areas.

Dain et al. (91) have utilized thin layer chromatography for the separation of gangliosides from canine, bovine, ovine, and human origin into three components. Bovine brain gangliosides were fractionated by silicic acid column chromatography. In addition the column chromatographic fractionation of gangliosides has been employed for the preparative isolation of four fractions. Molecular ratios of the residues in each of the fractions indicated non-homogeneous preparations. 
Thin layer silicic acid chromatography, first described by Stahl (92), has been widely employed for the identification and resolution of gangliosides. Individual preferences and purposes have resulted in the utilization of numerous solvent systems and modifications, One of the solvents most widely used for the resolution of gangliosides is chloroform/methanol/water, 60:35:8, reported by Wagner et al. (93). Johnson at al. (94) have noted the presence of five gangliosides in a human ganglioside preparation. Stmilar results were reported by Trams and Lauter (37) with gangliosides isolated by the tetrahydrofuran extraction procedure. Müldner et al. (115) have resolved on TIC a ganglioside preparation into eight components.

Svennerholm (95) has briefly described the composition of five gangliosides; one of these was reported to be the major ganglioside occurring in Tay-Sachs' disease.

The above discussion has not necessarily followed the chronological sequence in which the numerous reports were published. An attempt was made to provide an order of contimuity in these investigations aimed at determining the number of gangliosides. A summary of these investigations indicates that the various normal ganglioside preparations consist of at least four clearly chromatographically resolvable gangliosides. The Tay-Sachs' ganglioside represents a fifth ganglioside which may or may not be distinguishable in the chromatographic separation of normal gangliosides. 


\section{Structure of Gangliosides}

Miost of the views related to the composition and number of components in ganglioside preparations were elaborated in the previous section. The failure to recognize at this time a basic structural formula for gangliosides has been due in large part to the wealth of conflicting data present in the literature (see Table 1). The accurate derivation of structural formulae for these very closely related molecules is greatly dependent upon the ability to isolate chromatographically homogeneous gangliosides.

The earliest formula of brain ganglioside was suggested by Klenk and Lauenstein (86) (Figure 2). Their formula for the compound, ganglioside, was suggested shortly after the isolation of chondrosamine (galactosamine) by Blix et al. (II).

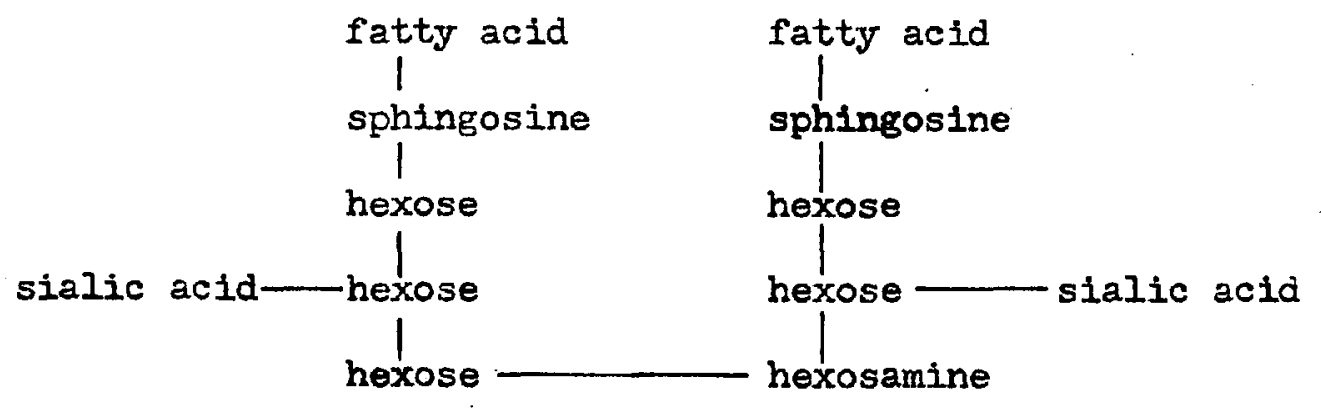

Figure 2.--Ganglioside by Kenk and Iauenstein

Svennerholm (36) proposed a formula for one of the fractions eluted from a cellulose column on the basis of compositional analyses as follows:

fatty acid-sphingosine-hexose-hexose-hexosamine-sialic acid

Bogoch (26) subjected an ox brain ganglioside preparation to selective degradative hydrolysis and identified the dialyzable 
TABLE 1

CARBOHYDRATE COMTOSITION OF GANGLIOSIDES

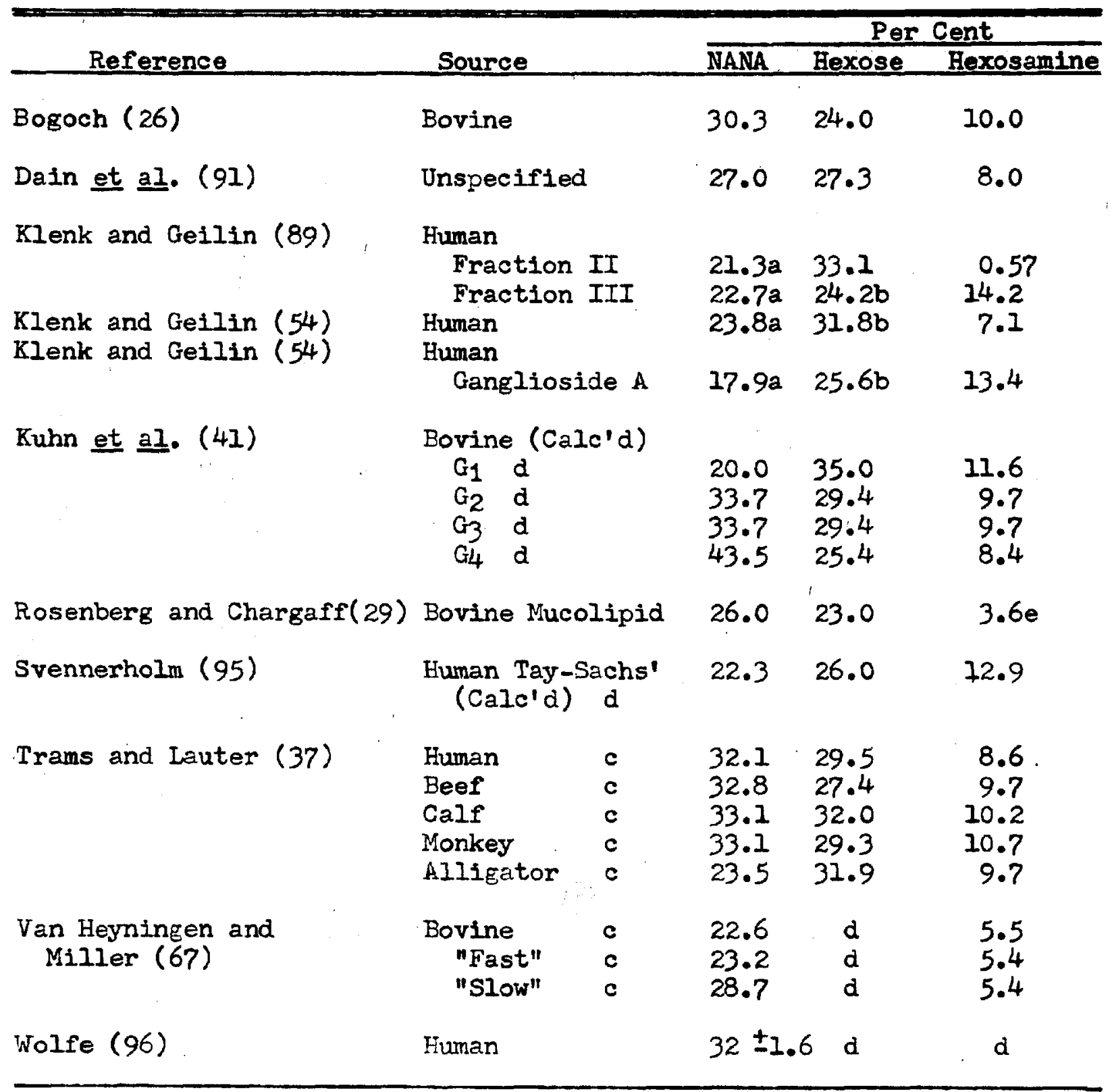

a NANA value converted from values reported as methoxymeuraminic aoid

b Hexose value determined by subtracting hexosamine value from total reducing sugar assuming equal reducing ability

c Per cent values deternined from umole/mg. values in literature;

Molecular weights used: NANA 309; Hexose 180; Hexosamine 179

d Values not reported

e Calculated from $\mathrm{N}$-acetylgalactosamine 
products, which included sialic acid-galactosamine, galactosaminegalactose-glucose, glucose-sphingosine, a glucocerebroside, and a hexodicerebroside. These data together with compositional analyses and molecular weight determinations of the ganglioside In aqueous solution led to the following formula for the repeating unit in the macromolecular ganglioside (Figure 3).

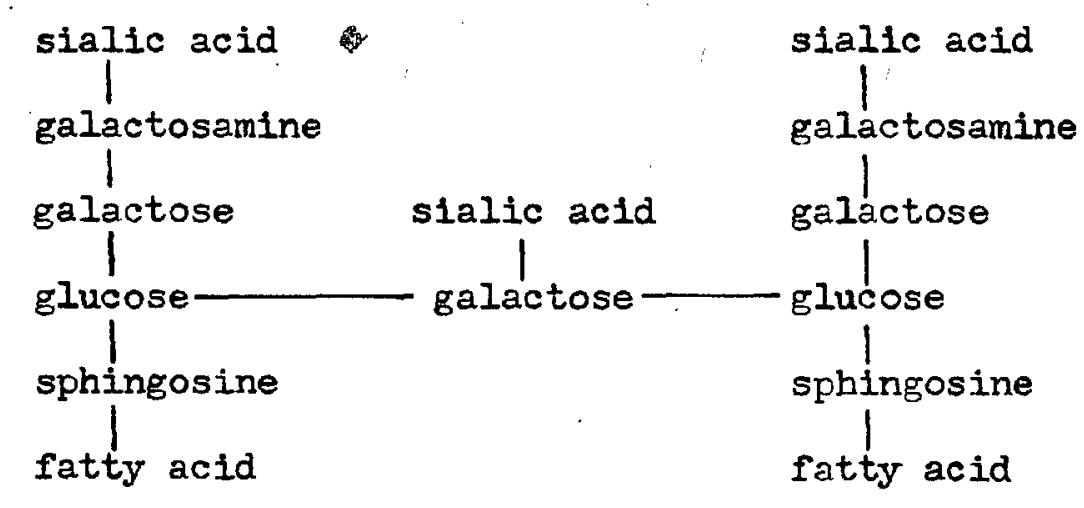

Molecular weight of unit: 3314

Figure 3.--Repeating Unit of Ganglioside

In an effort to provide a structural formula for ganglioside which would agree with molecular weight and equivalent weight data, Klenk and Geilin (40) subjected a ganglioside preparation to methylation, periodate oxidation, and acid hydrolysis. Exhaustive methylation followed by acid hydrolysis yielded 2,3,4,6-tetramethylgalactopyranose, 2,3,6-trimethyl-glucopyranose, 2,4,6-trimethylgalactopyranose, 2,6-dimethyl-galactopyranose, and 4,6-dimethyl2-deoxy-2-amino-galactopyranose. Periodate oxidation destroyed only glucose. One of two disaccharides isolated from the acid hydrolysate contained galactosamine and galactose. Borohydride reduction followed by acid hydrolysis and paper chromatography placed the 
reducing end of the disaccharide in the galactose residue. A chromatographic similarity was noted with this disaccharide and 3(-2-acetamido-2-deoxygalactopyranosido)-galactose. The second disaccharide was reported only to contain galactose.

On the basis of these data structures were proposed for a hexosamine-containing ganglioside (Formula I) and a hexosaminefree ganglioside (Formula II) (Figure 4). Unlike the former, the structure for the hexosamine-free ganglioside contained a branched oligosaccharide unit. Klenk and Geilin have continued to note the presence of a hexosamine-free ganglioside. An acetylated ganglioside component with this characteristic was eluted from a cation exchange column (Fraction II) (89); chromatography of this fraction on a silicic acid column resolved it into two components (Ganglioside C and Ganglioside D), both of which contained essentially no hexosamine (54).

The chromatographically homogeneous Ganglioside A which was eluted from the silicic acid column contained equimolar quantities of sphingosine, stearic acid, glucose, galactose, N-acetyl-galactosamine, and N-acetylneuraminic acid (NANA). Although no structure was proposed for Ganglioside A, the evidence obtained from methylation, periodate oxidation, and compositional analyses suggested a structural formula identical to Formula I (Figure 4). This component was the fastest moving of the four gangliosides in the solvent system used.

The ganglioside originally designated $G_{2.0}$ by Kubn (88) contained equimolar amounts of sphingosine, fatty acid, glucose. 


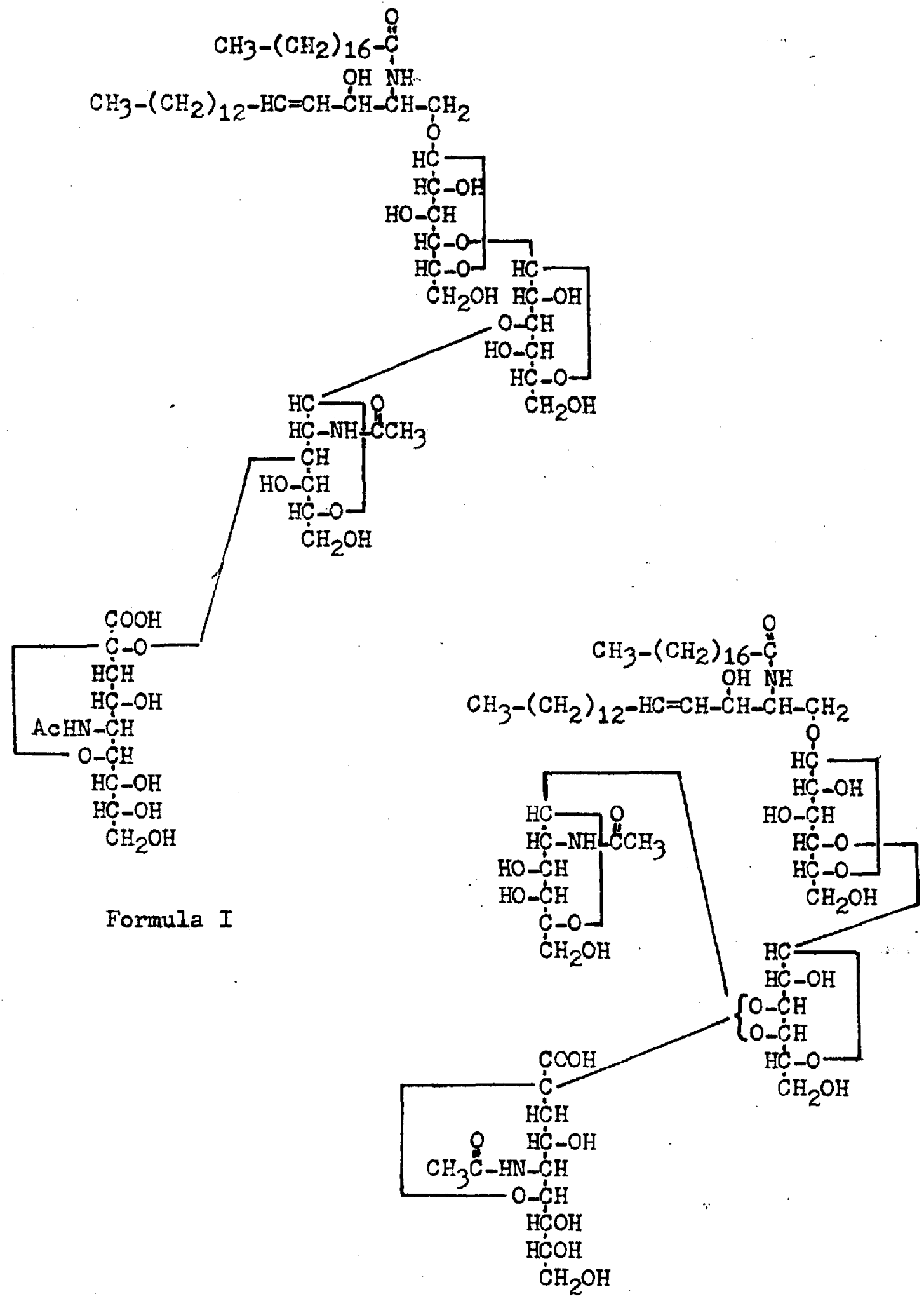

Formula II

Figure 4.--Structures for Gangliosides (Klenk and Geilin (40)) 
galactose, galactosamine, and NANA; an equivalent weight of $1380 \pm_{20}$ was determined by titration (20). Mild methylation procedures. which resulted in less than maximal theoretical methoxy content in the product yielded upon hydrolysis 2,6 dimethyl-glucopyranose and 2,4,6-trimethyl-galactopyranose. More vigorous methylation conditions yielded 2,3,6-trimethyl-glucopyranose and 2,3,4,6-tetramethyl-galactopyranose. Additional methylation products were 2,6-dimethyl-galactopyranose and 3,4,6-trimethyl-2-deoxy-2-aminogalactopyranose (48). Egge (48) considered the tetramethyl-galactose and the trimethyl-glucose to be artifacts caused by the alkaline lability of the C-3 glycosidic bond at both galactose and glucose units during the methylation. Acetolysis of this ganglioside followed by controlled acid hydrolysis (glacial acetic acid in acetic anhydride to which $\mathrm{H}_{2} \mathrm{SO}_{4}$ was added) yielded several oligosaccharides, which were isolated and characterized (Table 2).

TABLE 2

OLIGOSACCHARIDES FROM ACETOLYSIS BY KUHN TT AL. (20)

\begin{tabular}{|c|c|c|}
\hline Number of Units & Units Present & $\begin{array}{l}\text { Reducing } \\
\text { Group }\end{array}$ \\
\hline Tetrasaccharide & NANA, N-Ac-gaINH $2, g a l, g l u$ & Glucose \\
\hline Trisaccharide & $\mathrm{N}-\mathrm{Ac}-\mathrm{galNH} 2, \mathrm{gal}, \mathrm{glu}$ & Glucose \\
\hline Trisaccharide & NANA, gal, glu & Glucose \\
\hline Disaccharide & gal, glu & Glucose \\
\hline Disaccharide & $\mathrm{N}-\mathrm{Ac}-\mathrm{galNH_{2 }}$, glu & Glucose \\
\hline
\end{tabular}

abbreviations: $\quad \mathrm{NANA}=\mathrm{N}-a c e t y l$ neuraminic acid; $\mathrm{N}-\mathrm{Ac}-\mathrm{galNH} 2=\mathrm{N}-a c e t y \mathrm{I}-$ galactosamine; gal=galactose; gluaglucose This disacchartde was found to be chromatographically identical to lactose. 
The two glucose containing disaccharides noted in Table 2 contained their reducing function in the glucose residue. This fact indicated a branching. in the oligosaccharide segment at the glucose unit in Ganglioside $G_{2}$ (Figure 5).

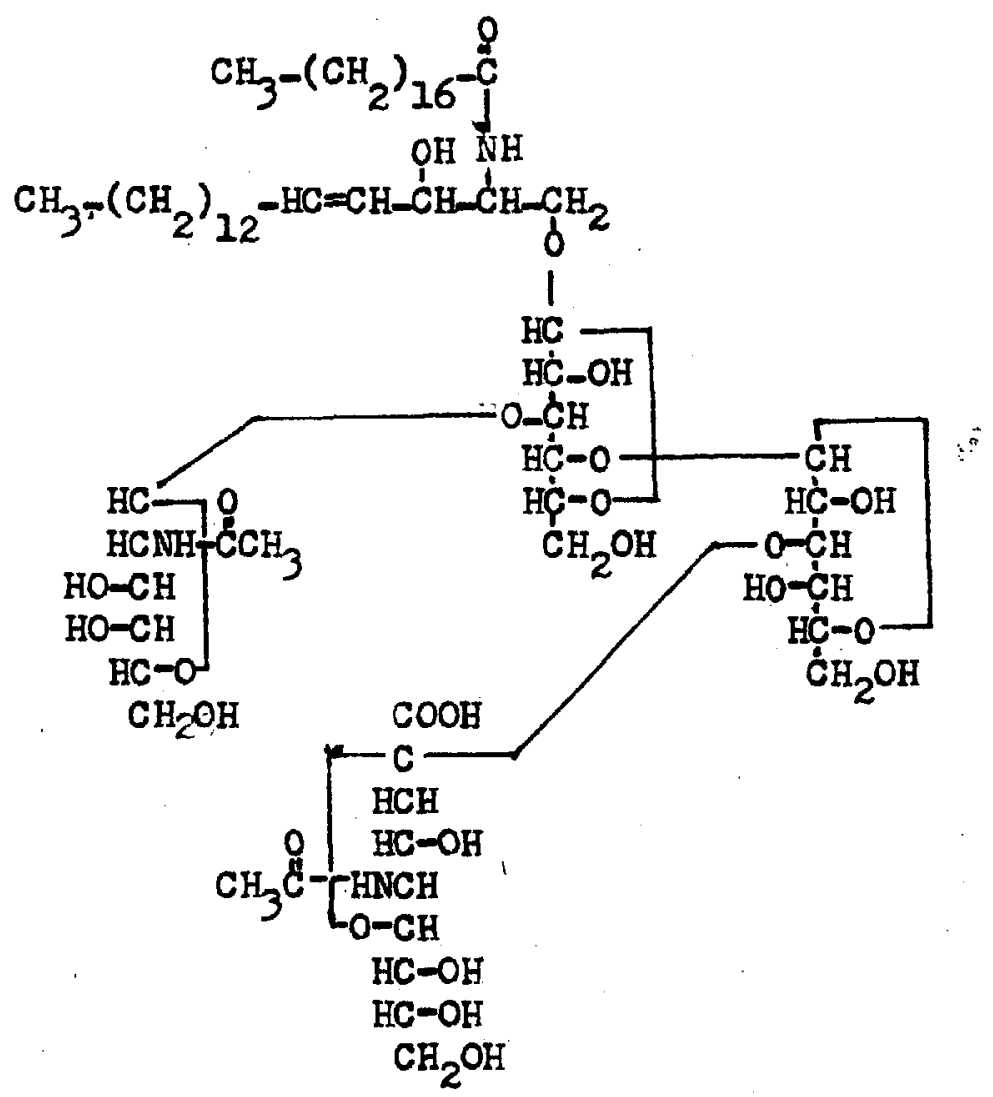

Figure 5.--Ganglioside $G_{2}$ (Kubn et al. (20))

The molecular composttion for the four bovine gangliosides which were crystallized by Kuhn et 2l. (41) has been reported (Table 3). The composition of hexose and hexosamine in a ratio of 3:1 in each ganglioside required a revision of the previously described $G_{2}$ to agree with the composition of $G_{1}$ in the table. Analytical data to substantiate this proposal have not been reported. 
TABLE 3

COMPOSITION OF BOVINE GANGLIOSIDES

(Kuhn et al. (4I))

\begin{tabular}{lllll}
\hline \hline & $G_{1}$ & $G_{2}$ & $G_{3}$ & $G_{4}$ \\
\hline Fatty Acid (Stearic) & 1 & 1 & 1 & 1 \\
Sphingosine & 1 & 1 & 1 & 1 \\
Glucose & 1 & 1 & 1 & 1 \\
Galactose & 2 & 2 & 2 & 2 \\
N-Acetylgalactosamine & 1 & 1 & 1 & 1 \\
N-Acetylneuraminic Acid & 1 & 2 & 2 & 3 \\
\hline
\end{tabular}

The gangliosides are listed in order of decreasing chromatographically mobility, i,e., $G_{1}$ was the fastest moving ganglioside.

The effect of neuraminidase or weak acid upon the trisialoganglioside $G_{4}$ and the disialogangliosides $G_{2}$ and $G_{3}$ was noted by Kuhn et al. (41) as follows:

$$
\mathrm{G}_{2} \longrightarrow \mathrm{G}_{1} \longleftarrow \mathrm{G}_{3} \longleftarrow \mathrm{G}_{4}
$$

The source of the neuraminidase and the relative enzymic reaction rates with $G_{2}$ and $G_{3}$ were not reported.

Rosenberg and Chargaff (97) have subjected their bovine mucolipid preparation to periodate oxidation. This preparation, which had a hexose to sialic acid ratio of $3: 2$, consumed 1.7 moles of periodate per mole of mucolipid-bound sialic acid. A resultant loss of approximately one-half of the sialic acid in the intact mucolipid indicated that only one-half of the sialic acid residues was linked terminally. Incubation of the mucolipid with $0.1 \mathrm{~N} \mathrm{H}_{2} \mathrm{SO}_{4}$ at $80^{\circ}$ for two hours essentially completely liberated the sialic acid without accompanying loss of hexose or hexosamine. The hydrolysate 
obtained reduced 3.2 moles periodate per mole sialic acid. Free NANA consumed 2.7 moles periodate in contrast with the 2.0 moles which theoretically should be consumed. This additional 0.5 moles of periodate consumed in the mucolipld hydrolysate accounted for a loss of one-third of the hexose content. Since the asialomucolipid reduced only 0.5 moles periodate, the authors suggested that statistically one of three hexose units carried both sialic acid molecules, "probably as a $2 \rightarrow 8$ linked dimer".

A second inference of a possible sialic acid dimer in ox brain gangliosides was noted by Wolfe (96) who found that only two-thirds of the carboxyl groups of the NANA residues were titratable. The differential release of NANA residues upon acid hydrolysis also indicated that not all of the NaNA residues are terminal units as has long been suggested.

The bovine mucolipid was subjected to exhaustive methylation by Karkas and Chargaff (98). After hydrolysis the methylated sugars were identified as 2,6-dimethyl-galactopyranose, 2,4,6trimethyl-galactopyranose, 2,3,4,6-tetramethyl-galactopyranose, and-but not fully identified--2,4,6-trimethyl-glucopyranose in the ratio of $7: 3: 3: 2$. No mention was made of the methylated hexosamine derivative. If complete methylation was achieved, the relatively large quantity of the dimethyl-galactose indicates a significant degree of branching in the oligosaccharide unit.

Trams and Lauter (37) have also suggested that gangliosides from some species may have a branched chain in the molecule. This proposal was based upon the differential liberation of sialic acid 
from the gangliosides by acid hydrolysis and by neuraminidase activity in time course studies. Approximately two-thirds of the NANA was released from the various ganglioside preparations by neuraminidase from influenza virus.

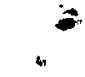

The four normally occurring ganglioside species isolated by Svennerholm (95) were reported to consist of a monosialoganglioside and three distalogangliosides $(a, b$, and $c$, in order of decreasing chromatographic mobility). All four species were converted by acid hydrolysis to one asialoganglioside which contained ceramide, glucose, galactose, and galactosamine in the ratio of $1: 1: 2: 1$. Characterization of this asialoganglioside as a straight chain molecule was based principally upon observations: (I) the identification of two disaccharides containing galactosamine and galactose which upon borohydride reduction and subsequent hydrolysis yielded galactose, galactosamine, dulcitol, and galactosaminol, (2) a tetrasaccharide consisting of glucose, two moles of galactose and galactosamine, and (3) the methylation products obtained by Klenk and Geilin (83), namely 2,3,6-trimethyl-glucopyranose, 2,4,6-trimethyl-galactopyranose, and 4,6-dimethyl-2-deoxy-2-amino-galactopyranose. The first two results indicated a carbohydrate sequence of glucose-galactose-galactosamine-galactose with the glucose unit. no doubt, linked glycosidically to the sphingosine as in a glucocerebroside. Although the methylation data of Klenk may not have been confirmed, its utilization by Svennerholm completed the information necessary for the structural formula of the asialogangIioside (Figure 6). The site of the glycosidic bond linking sialic 


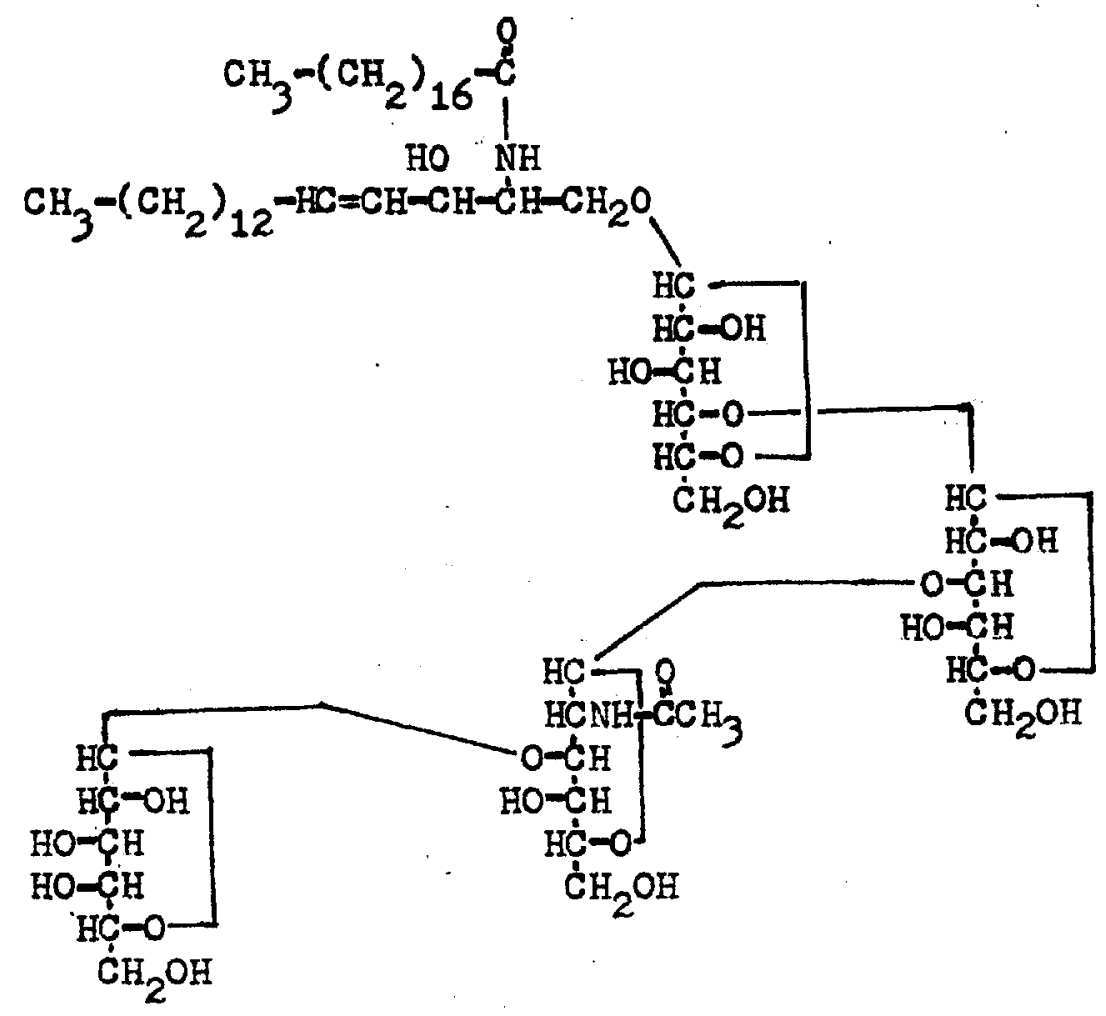

Figure 6.--Asialoganglioside of Svennerholm (95)

acid to the above structure was not proposed either for the monosialoganglioside or for the three disialogangliosides. The presence of the 2,4,6-trimethyl-galactose as the sole methylated derivative of galactose cited by Svennerholm would indicate the site of attachment of the sialic acid molecule in the monosialoganglioside at the C-3 position of the terminal galactose unit. The second sialic acid residues in the disialogangliosides $a, b$, and $c$ of Svennerholm (95) are susceptible to neuraminidase. The order of their reactivity was reported as csasb.

A structure was also proposed by Svennerholm for the component which accounted for 90 per cent of the total ganglioside fraction of a Tay-Sachs' brain. Equimolar quantities of ceramide, glucose, 
galactose, galactosamine, and NANA make up the composition of this abnormal ganglioside. This molecule lacked the terminal galactose unit that was present in the normal monosialoganglioside (Figure 6). The site of attachment of the sialic acid molecule in this abnomal ganglioside was neither indicated nor proposed. This asialo portion of the Tay-Sachs' ganglioside is identical to the corresponding segment of Klenk's hexosamine-containing ganglioside (Formula I) and possibly Ganglioside A. The latter has the sialic acid residue linked glycosidically to the C-3 position of the N-acetylgalactosamine.

A large segment of the information related to the structural composition of gangliosides in general, and the individual gangliosides in particular, has been noted in this section. The extent of the conflicting data which was noted in the introduction to this section now must be quite evident. The possibility of juxtaposing this entire array of often incongruous data into a solution which embraces all aspects of ganglioside structure seems quite remote. Some degree of orderliness may be salvaged in this respect by summarizing categorically the results of the numerous investigations that have thus far received attention.

This incongruity is probably most apparent in the sequence of carbohydrate residues in the oligosaccharide section of gangliosides. Several oligosaccharides consisting of various carbohydrates have been identified from the acid hydrolysates of a number of ganglioside preparations. These oligosaccharides are listed in Table 4. 
TABLE 4

OLIGOSACCHARIDES RELEASED FROM GANGLIOSIDES

DURING ACID HYDROLYSIS

\begin{tabular}{|c|c|c|c|}
\hline Reference & No. of Units & Units $^{a}$ & $\begin{array}{l}\text { Reducing } \\
\text { Group }\end{array}$ \\
\hline Bogoch (26) & $\begin{array}{l}\text { Disaccharide } \\
\text { Trisaccharide }\end{array}$ & $\begin{array}{l}\text { NANA, galNH, } \\
\operatorname{galNH}_{2}, \mathrm{gal}^{2} \text { glu }\end{array}$ & $\operatorname{galNH}_{2}$ \\
\hline $\begin{array}{l}\text { Klenk \& Geilin } \\
(40,89)\end{array}$ & $\begin{array}{l}\text { Disaccharide } \\
\text { Disaccharide }\end{array}$ & $\begin{array}{l}\text { gal, } \operatorname{galNH}_{2} \\
\text { gal, gal }\end{array}$ & $\begin{array}{l}\text { gal } \\
\text { gal }\end{array}$ \\
\hline Kuhn' et al. (20) & $\begin{array}{l}\text { Tetrasaccharide } \\
\text { Trisaccharide } \\
\text { Trisaccharide } \\
\text { Disaccharidec } \\
\text { Disaccharide }\end{array}$ & $\begin{array}{l}\text { NANA, galNH, gal, glu } \\
\text { galNH, gal, glu } \\
\text { NANA, gal, glu }_{2} \\
\text { gal, glu } \\
\text { galNH, glu }\end{array}$ & $\begin{array}{l}\text { glu } \\
\text { glu } \\
\text { glu } \\
\text { glu } \\
\text { glu }\end{array}$ \\
\hline Svennerholm (95) & $\begin{array}{l}\text { Tetrasaccharide } \\
\text { Disaccharide } \\
\text { Disaccharide }\end{array}$ & $\begin{array}{l}\text { gal, gal, galNH } 2^{\prime} \text { glu } \\
\text { galNH } \text { gal }^{\prime} \text { galNH2 gal }\end{array}$ & $\begin{array}{l}\mathrm{glu} \\
\mathrm{galNH} \\
\operatorname{gal}\end{array}$ \\
\hline $\begin{array}{l}\text { Trams \& Lauter } \\
\text { (37) }\end{array}$ & Disaccharide & gal, glu & gIu \\
\hline
\end{tabular}

abbreviations: $\mathrm{NANA}=\mathrm{N}$-acetylneuraminic acid; galNH $2=\mathrm{N}-a c e t y l-$ galactosamine; gal=galactose; glu=glucose

beducing group where positively identified

CThis disaccharide was identified as lactose. 
The results of the methylation experiments are also reviewed (Table 5). The variation in the resulting methylated products may be due either to incomplete methylation or to alteration of the molecule during methylation, which exposes additional sites for methylation. A comment by Egge (48) which was cited earlier suggested that tetramethyl-galactose and trimethyl-glucose were artifacts caused by the alkaline lability of the glycosidic bond at the $c-3$ position of each of these residues.

A summary of the periodate oxidation data is not as extensive. Klenk and Geilin $(40,54,83,89)$ have reported the destruction of glucose by periodate in the total ganglioside preparation and in their isolated fractions. The detection of partial destruction of other residues except NANA in the unfractionated gangliosides would be difficult. -Rosenberg and Chargaff (97) have reported the loss of approximately one-half of NANA in the intact bovine mucolipid through periodate oxidation.

A sizeable percentage of the ganglioside-bound sialic acid is susceptible to cleavage by neuraminidese. Most reports concur in this finding. The glycosidic bond which links the remaining sialic acid residue to the monosialoganglioside(s) is apparently not sensitive to enzymatic cleavage. It is reasonable to assune that the sialic acid residues remaining after neuraminidase treatment are present in the basic monosialoganglioside molecule. This also indicates that at least two different bonds are present by which sialic acid residues are linked to the disialogangliosides. 
TABLE 5

CARBOHYDRATE DERTVATIVES IDENTIFTED FROM

GANGLIOSIDE METHYLATION EXPERTMENTS

\begin{tabular}{|c|c|c|c|}
\hline Methyl Sugar Derivative & \multicolumn{3}{|c|}{$\begin{array}{r}\text { Reference }^{a} \\
\infty\end{array}$} \\
\hline $2,3,4,6$-Tetramethyl-Galactose ${ }^{b}$ & K\&E & K\&G & $K 8 C$ \\
\hline 2,4,6-Trimethyl-Salactose & $K \& E$ & K\&Ot & $\mathrm{K} 8 \mathrm{C}$ \\
\hline 2,6-Dimethyl-Galactose & $K \& E$ & & K\&EC \\
\hline $2,3,6-$ Trimethyl-Glucose $^{b}$ & $K \& E E$ & $K \& G$ & \\
\hline $2,4,6-\operatorname{Tr}$ imethyl-Glucose $e^{c}$ & & & $\mathrm{~K} \& \mathrm{C}$ \\
\hline 2,6-Direthyl-Glucose & $\mathrm{K} \& \mathrm{E}$ & & \\
\hline 3,4,6-Trimethyl-Galactosamine & $\mathrm{KLEE}$ & & \\
\hline 4,6-Dimethyl-Galactosamine & & $K \& G$ & \\
\hline
\end{tabular}

a Symbols used: K\&E Kuhn and Egge" (20,48)

K\&G Klenk and Geilin (40)

KeC Karkas and Chargaff (98)

bonsidered as artifacts by Egge (48)

$c_{\text {Not fully }}$ Identified 
Although the relative neuraminidase reactivity of Kuhn's disaccharides $G_{2}$ and $G_{3}$ was not reported, the reaction rates of these gangliosides were strikingly similar with respect to the gangliosides of Svennerholm (95) in relation to their chromatographie mobility. In both cases the slowest moving ganglioside (trisialoganglioside $G_{4}$ and disacchartde $c$, respectively) were most reactive.

Glucose apparently occurs in the ganglioside molecule only as the carbohydrate residue proximal to the sphingosine, 1.e., in the form of a glucocerebroside. Recent evidence also infers that all sialic acid residues in gangliosides may not be terminal as was generally suspected.

Although the configuration of the glycosidic bonds illustrated in the figures are predominantly $\beta$, the configurations have not been established.

The problems which remain to be solved in the structure and composition of gangliosides are multiple. Indeed the problems which have been solved to the concordance of most competing investigators whose interests lie in this discipline are still too few. Improved methods of isolation of gangliosides and resolution of Individual gangliosides together with the observations and interpretations of previous experiences should gradually erase these Incongmities and effect an accurate solution. 


\section{EXPERTMENTAL}

(3)

\section{Analytical Methods}

N-AcetyIneuraminic Acid Determination

Ganglioside N-acetylneuraminic acid (NANA) was quantitatively determined by the resorcinol method of Svennerholm (77) as modified by Miettinen and Takki-Iuukkainen (78). Dichromatic readings were made at $580 \mathrm{mp}$ and $450 \mathrm{mp}$ to compensate for the interference of hexoses. NANA, which had been isolated from human plasma according to the method of Martensson et al. (99), was employed as a standard for each analysis. Molecular extinction coefficients were determined for NANA and galactose at these two wave lengths and were in agreement with those values already reported (81).

\section{Hexosamine Determination}

Hexosamine in ganglioside acid hydrolysates was quantitatively determined according to Method B of Svennerholm (100). N-acetylglucosamine (Nutritional Biochemicals Corporation, Cleveland, Ohio) was employed as a standard.

\section{Hexose Determination}

An anthrone assay (114) was utilized for the determination of ganglioside hexose. The anthrone reagent consisted of $80 \mathrm{mg}$. of recrystallized anthrone (101) in $10 \mathrm{ml}$. water and $100 \mathrm{ml}$. concentrated $\mathrm{B}_{2} \mathrm{SO}_{4}$. Five ml. of this reagent was added with mixing to two $\mathrm{ml}$. of an aqueous solution containing ganglioside or other 
sugars in an $18 \times 125 \mathrm{~mm}$. Pyrex test tube. The tubes were placed in a bolling water bath for $3 \frac{1}{2}$ minutes. Optical densities of the cooled solutions were read at 620 mp. Standard curves for galactose (Pfanstiehl Chemical Company, Waukegan, Illinois) and glucose (Nutritional Biochemicals Corporation, Cleveland, Ohio) were made in the range 0 to $200 \mathrm{\mu g}$. The molecular extinction coefficient of glucose was 1.3 times that of galactose. All hexose values were expressed as galactose equivalents. .

A phenol- $\mathrm{H}_{2} \mathrm{SO}_{4}$ assay (102) was employed for hexose determinations in ganglioside hydrolysates. This assay was much more sensitive than the anthrone assay. A second distinct advantage was that the molecular extinction coefficient of glucose was only 1.1 times that of galactose. All values for hexose in the hydrolysates were expressed as galactose equivalents.

Glucose Detemination

The glucose oxidase (Dade Reagents, Inc., Miamt, Florida) method of Saifer and Gerstenfeld (103) was employed in one experiment for the determination of glucose in a ganglioside hydrolysate. Sphingosine Determination

Ganglioside sphingosine was determined after hydrolysis by the spectrophotometric method of Trams and Lauter (104) in one experiment. Phosphorus Determination

Phosphorus in ganglioside preparations was determined according to Harris and Popat (105). 


\section{Infrared Analysis}

, A $2 \mathrm{mg}$. ganglioside sample was mixed with $400 \mathrm{mg}$. KBr in an agate mortar. The sample was pressed in a hydraulic press, and the spectrum of the resulting pellet was recorded on a Beckman IR- 4 infrared spectrophotometer. An infrared spectrum of NANA was obtained in a similar manner.

\section{Isolation of Gangliosides}

Human cerebral tissue specimens were obtained from the Department of Pathology soon after autopsy. Gray matter with minor amounts of contaminating white matter was homogenized with water and lyophilized.

In the initial isolation procedure gray matter was extracted with 95 per cent acetone and then dried under $\mathrm{N}_{2}$. The dried residue was extracted with methanol/chloroform, $2: 1$, in a Soxhlet extractor. The methanol/chloroform extract was evaporated under vacuum, and the resulting residue was subjected to a two tube, double-withdrawal distribution between the two phases of chloroform/methanol/0.1 per cent $\mathrm{NaCl}, 8: 4: 3(35)$. The two upper phases were combined. concentrated by rotary evaporation, dialyzed, and lyophilized. This residue was dissolved in water and passed through a Sephadex $50^{3}$ column $(30 \times 3 \mathrm{~cm}$.$) . Two resorcinol-positive peaks were eluted$ as has been described by McCluer et al. (81); the first or major peak was then lyophilized to yield a residue containing 12.4 per cent NANA by resorcinol. This residue was passed over a Dowex $50 \mathrm{~W}-\mathrm{X} 1$ (Ht)

3 Sephadex is a product of Pharmacia, Uppsala, Sweden. 
Ion exchange column, and the eluate was lyophilized. The NANA and phosphorus contents were 18 and 0.88 per cent respectively. It was noted that a dark band remained at the top of the ion exchange column.

The high phosphorus content prompted a revision in subsequent ganglioside isolations. Iyophilized human gray matter (297 gm. $)^{4}$ was extracted with 95 per cent acetone (1:10, weight/volume), for two hours at room temperature. After two subsequent acetone extractions for ten minutes each ( $1: 2$, original weight/volume), the acetone was filtered through a folded cheesecloth. Additional solvent was removed by firmly squeezing the residue in the cheesecloth. This residue was then subjected to ether extraction (1 liter) for six hours while standing in the cold room with gentle agitation. The supernate was decanted, additional ether ( $500 \mathrm{ml}$.) was added to the residue, and this extraction was continued for thirty minutes. The ether was filtered through cheesecloth as above. The residue was dried as thoroughly as possible under a gentle stream of $\mathrm{N}_{2}$ and subjected to extraction with methanol/ chloroform, 2:1, in a Soxhlet extractor for 24 hours.

The extract was concentrated by rotary evaporation and lyophilized. This residue was subjected to a four tube, doublewithdrawal solvent distribution between the two phases of chloroform/ methanol/0.1 per cent $\mathrm{NaCl}, 8: 4: 3$ (35). The combined upper phases were concentrated by rotary evaporation and dialyzed until the

4Values are given for one typical experiment 
conductivity in the dialysate approximated that of distilled water 5 . The dialyzed material was then passed through a Dowex 50W-X1 (Ht) ion exchange resin column, lyophilized and dried in a desiccator over $\mathrm{P}_{2} \mathrm{O}_{5}$. This procedure is outlined in Figure 7. The total yield was $2.0 \mathrm{gm}$. of a fluffy white material which had a NANA content of 28.4 per cent and 0.23 per cent phosphorus. Redistribution of the gangliosides between the two phases of the solvents noted above resulted in a product with a lower phosphorus content and an increased NANA content.

\section{Chromatographic Procedures}

Paper Chromatographic Procedures

Standard chromatographic techniques were employed throughout this investigation (106). All solvent systems employed in this and in other chromatographic procedures were based on volume/ volume ratios.

Thin Iayer Chromatographic Procedures

Techniques employed in thin layer chromatography (TIC) have been described by Mangold (107) in his thorough review of the materials and methodology of this recently developed chromatographic innovation. In this investigation a slurry of $30 \mathrm{gm}$. of Silica Gel $\mathrm{G}^{6}$ in $60 \mathrm{ml}$. water was applied to clean glass plates $(20 \times 20 \mathrm{~cm}$.) with the aid of an adjustable applicator (Model 59 . which spread a

The dialysate produced a resistance of 70,000 ohms whereas distilled water gave a resistance of 75,000 ohms. Double distilled water had a resistance of 115,000 ohms.

6E. Merck A. G., Darmstadt, Germany. American Distributors: Brinkmann Instruments, Inc., Great Neck, N. Y. 


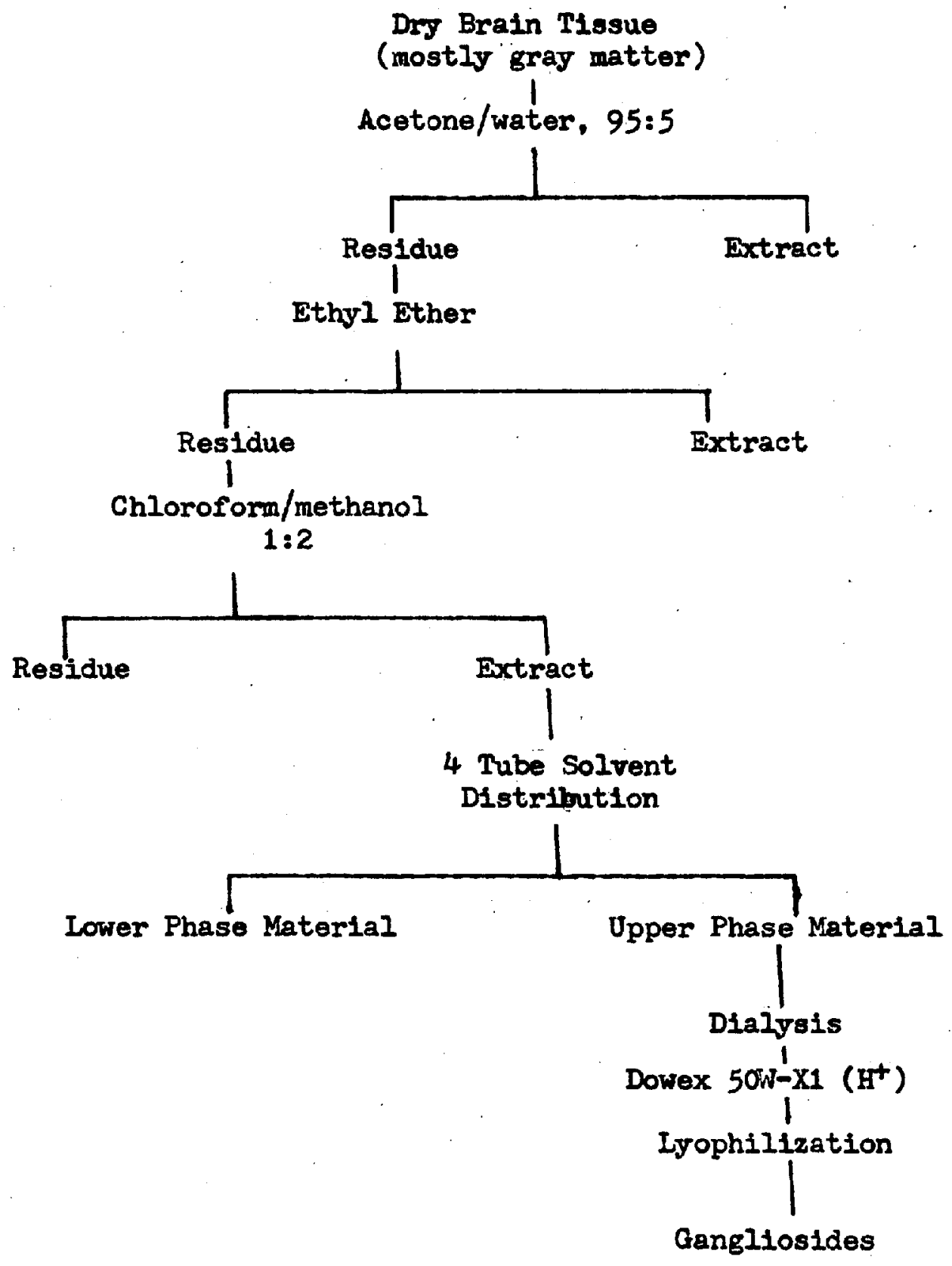

Figure 7.--Preparation of Gangliosides from Normal Sdult Human Brain T1ssue. 
uniformly thick layer of the silicic acid adsorbent on the plate. Except for attempts at preparative isolation of gangliosides by TIC, layers of $250 \mu$ were used. The plates were air-dried for 10-20 minutes, activated by heating at $105-110^{\circ}$ for 30 minutes, and placed in a desiccator over activated silica gel.

Preparatory to spotting on TIC plates, gangliosides were dissolved in small amounts of chloroform/methanol (approximately 1.1). The chloroform/methanol solution was applied to the adsorbent layer with micro pipettes $(5-50 \mu \mathrm{\mu l})$ in $1 \mathrm{~cm}$. streaks or in a single spot. Rapid evaporation of this solvent was facilitated by a gentle stream of cool air from a hair dryer. The plates, after being spotted, were placed in a chamber in which the atmosphere had been pre-equilibrated with the desired solvent. The solvent was" usually allowed to move up the entire plate surface before the plate was removed, air-dried, and sprayed with the desired reagent.

Utilization of TIC for the preparative isolation of gangliosides required slightly different conditions. Layers of $500 \mu$ and $750 \mu$ were spread with the adjustable applicator. The ganglioside was often spotted in 2-3 cm. streaks at the line of origin, which was $1.5 \mathrm{~cm}$. from the bottom of the plate. Anasil $\mathrm{S}^{7}$ and Anasil $\mathrm{B}^{7}$, and Kieselguhr ${ }^{6}$ were other silicic acid adsorbents employed for the TIC of gangliosides. Silica Gel $\mathrm{G}^{6}$ produced the most favorable results.

PSilicic acid products of the Analytical Engineering Laboratories, Inc., Hamden, Connecticut 


\section{Column Ghromatographic Proceduros}

Preparation of Silicic Acid for Column Chromatography. Silicic acid powder ${ }^{8}$ passing 80 mesh screen was washed with methanol ( $2 \mathrm{ml}$. per $\left.g_{*}\right)$, filtered through a Bilchner funnel, air-dried at room temperature, and then activated at $65-67^{\circ}$ for sixteen hours.

The adsorptive activity of the silicic acid was determined by the method of Hernandez et al. (108). An activity of IIb was established by the chromatographic resolution of Sudan yellow and p-methoxy-azobenzene. This silicic acid was used only in two columns. As the techniques of packing, elution and fraction analysis varied in these two columns, these conditions will be dealt with individually when the columns are described.

Preparation of Anasil $\mathbf{S}$ Columns. Although Anasil $S$ has been principally designed and publicized for use as an adsorbent in TIC, it was used here as a column adsorbent for the chromatographic separation of gangliosides.

Except for one instance when the Anasil was activated for thirty minutes at $115^{\circ}$. Anasil was taken directly from its container for packing in the column. A glass wool pad was first placed at the bottom of a clean glass column (often a $25 \mathrm{ml}$, or $50 \mathrm{ml}$. buret). Anasil was then added to the column in small amounts and packed dry by vibration and tamping. Ganglioside was mixed dry with a small amount of Anasil, placed on the column in as thin a band as possible, and an additional layer of Anasil was

8Mallinckrodt Chemical Works, St. Louis, Missouri, suitable for chromatographic analysis 
added. A glass wool pad concluded the packing of the column. The eluting solvents, flow rates, and volume, collection, and analysis of fractions will be discussed with each of the columns.

Acid Hydrolysis Procedures in Ganglioside Analyses Conditions for Hexosamine and Hexose fnalyses

Hexosamine. Standard hydrolysis conditions were established by subjecting a ganglioside preparation and a standard N-acetylglucosamine standard to hydrolysis in $2 \mathrm{~N} \mathrm{HCl}$ and by determining the hexosamine values as a function of hydrolysis time. Maximum values were noted at 18 hours. Approximately one mg. of ganglioside was employed for hexosamine analysis. Total reaction volume was about two $\mathrm{ml}$. for each hydrolysis, and each flask was flushed out with $\mathrm{N}_{2}$ before hydrolyzing in $2 \mathrm{~N} \mathrm{HCl}$ for 18 hours at $100^{\circ}$. The hexosamine content for one of the gangliosides isolated (FM) was increased by hydrolyzing in $5 \mathrm{~N} \mathrm{HCl}$ for seven hours.

Hexase. Hexose determinations were initialiy made on the 18 hour HCl hydrolysates. However, a 20 per cent loss of glacose was noted under these hydrolysis conditions although little loss of galactose was detected. Iittle or no loss of either of the hexoses was detected after hydrolysis for seven hours in $2 \mathrm{~N} \mathrm{H}_{2} \mathrm{SO}_{4}$. Hexose content of a ganglioside sample subjected to hydrolysis in $2 \mathrm{~N} \mathrm{H}_{2} \mathrm{SO}_{4}$ was maximal after 5-7 hours. Gangliosides were hydrolyzed for seven hours in $2 \mathrm{~N} \mathrm{H}_{2} \mathrm{SO}_{4}$ except for ganglioside $\mathrm{FM}$, which gave higher hexose values after hydrolysis for five hours in $3 \mathrm{~N} \mathrm{H}_{2} \mathrm{SO}_{4}$. In all cases $0.6 \mathrm{mg}$. was sufficient for hexose analyses by the phenol $/ \mathrm{H}_{2} \mathrm{SO}_{4}$ assay. 
Determination of Hexose Ratios

In order to maintain identical hydrolysis conditions, all ganglioside samples were hydrolyzed in $3 \mathrm{~N} \mathrm{H}_{2} \mathrm{SO}_{4}$ for 5-6 hours for the determination of the galactose/glucose ratio. The acid hydrolysates were neutralized with Dowex $1-\mathrm{X}^{8}\left(\mathrm{CO}_{3}=\right)$, concentrated on a rotary evaporator, spotted on Whatman No. I paper, and irrigated with the organic phase of benzene/n-butanol/pyridine/ water, $1: 5: 3: 3$ (117), for 40-48 hours. A more distinct separation of the galactose and glucose areas was noted if no equilibration was permitted after the chromatogram was introduced into the chromatographic chamber. A reference spot containing glucose and galactose was also spotted. After irrigation the chromatogram was air-dried, the reference strip was cut off and developed with a silver nitrate dip reagent (118). The staining areas on the reference strip were noted and used to mark off the corresponding areas on the sample strip before the reference strip was dipped in the sodium thiosulfate "fixer". These areas were cut out along with a blank area of similar size, cut into very small pieces, and placed in a filter beaker. Similar amounts of water were added to each beaker, the containers allowed to stand overnight or agitated for a shorter period of time and then filtered. Duplicate aliquots of the filtrate were then subjected to the phenol $/ \mathrm{H}_{2} \mathrm{SO}_{4}$ hexose assay, and the amounts of each hexose were determined from the slopes of the standard curves. Solutions containing known galactose to glucose ratios of $0.95,0.95$, and 0.47 were subjected to similar hydrolysis conditions and analyzed 
as above. The ratios deterinined were 1.0, 0.93, and 0.42 , respectively.

\section{Chromatographic Fractionation of Gangliosides on Silicic Acid Columns}

\section{Column I}

Silicic acid with activity noted above was dry-packed by tamping and vibration to provide a column of dimensions $27 \times 2.5 \mathrm{~cm}$. Ganglioside (750 mg.) from the initial isolation procedure (18 per cent NANA and 0.88 per cent phosphorus) was dissolved in a small volume of chloroform/methanol, 3:1, and applied with a pipette to the column. The initial solvent, chloroform/methanol, 3:1, was followed by chloroform/methanol mixtures of $2: 1,3: 2,1: 1,1: 2$, and methanol. Fractions $(15 \mathrm{ml}$.) were collected with the aid of a fraction collector at an average flow rate of $1 \mathrm{ml}$. per minute. Aliquots from alternate fractions were removed, dried under a gentle stream of $\mathrm{N}_{2}$, and analyzed for NANA by the resorcinol assay and for hexose by the anthrone assay. Elution of gangliosides as identified by the resorcinol assay is shown in Figure 8. Tubes 1-8 gave a positive, but cloudy, anthrone reaction and a negative resorcinol reaction. The solvent from these pooled fractions was removed by rotary evaporation, and the residue in the flask was swirled in a small amount of ether. Ether soluble material (126 mg.) contained 2.9 per cent phosphorus. Material which was not solubilized by this procedure ( $50 \mathrm{mg}$. ) contained 2.3 per cent phosphorus. These samples were not subjected to further analyses as the high phosphorus content indicated that they were primarily phospholipids. 


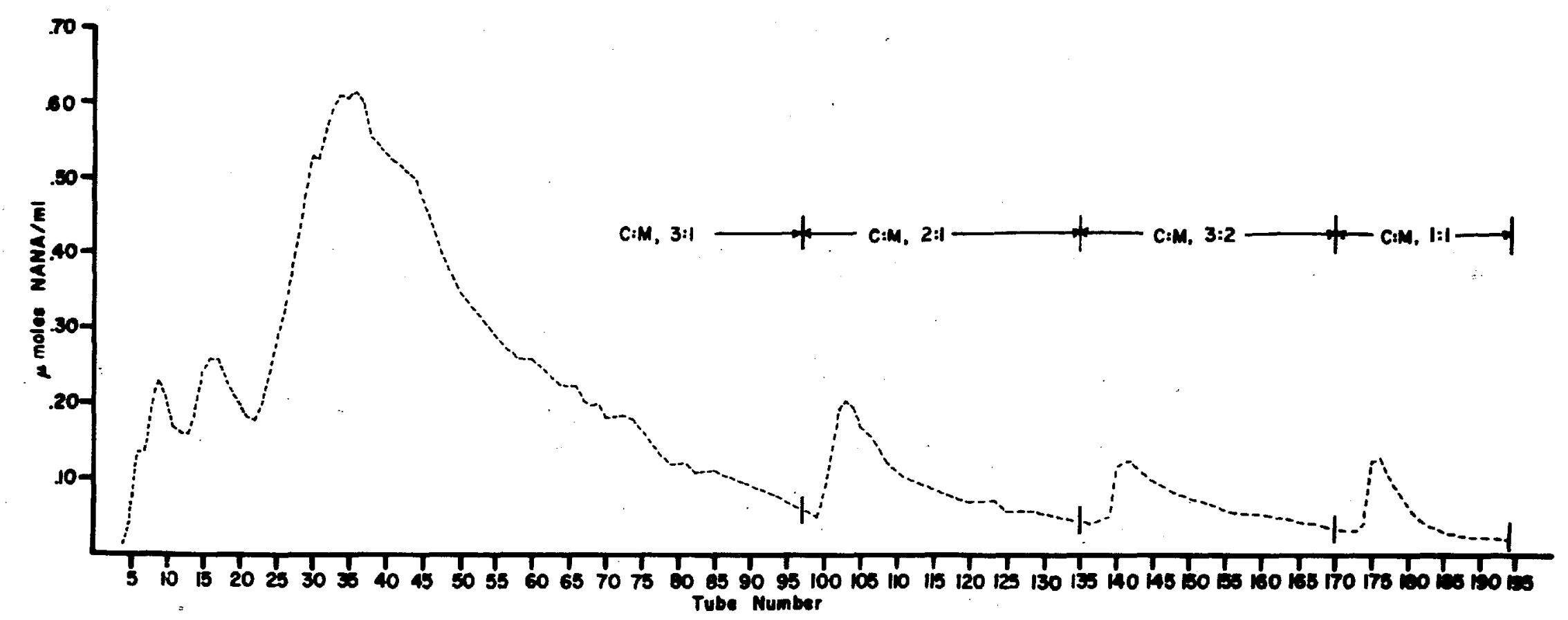

Figure 8.--Ganglioside Elution from Column I 
Fractions comprising the large resorcinol peak eluted with chloroform/methanol, 3:1, (Figure 8) were pooled and the solvent removed by evaporation. This residue (352 mg.) containing 0.50 per cent phosphorus was dissolved in fresh lower phase of the Folch solvents, chloroform/methanol/0.1 per cent NaCl, 8:4:3 (35). and subjected to a two tube double-withdrawal solvent distribution. After dialysis and lyophilization of the combined upper phases, the residue containing 0.23 per cent phosphorus was dissolved in upper phase and extracted with four fresh lower phases of Folch solvent. The upper phase was dialyzed and then lyophilized yielding a product designated Fraction 41 with 0.10 per cent phosphorus. A considerable loss of gangliosides was demonstrated in the lower phases which had not been washed with additional fresh upper phase.

Fractions comprising a single peak were pooled, the solvent removed, and the residues weighed. Since the quantity in each of the peaks eluted after the large resorcinol peak was insufficient to analyze individualjy, all the material eluted after chloroform/ methanol, 3:1 (tube 97), was pooled. This residue (70 mg.) was designated Fraction \#2.

Both of these ganglioside fractions were chromatographed on acetic acid-washed Whatman No. 3 paper. The solvent systems and conditions included:

(a) n-butanol/pyridine/water, 3:3:1 (descending)

(b) n-butanol/pyridine/water, 6:5:4 (ascending) (20)

(c) n-butanol/acetic acid/water, 4:1:5 (ascending) (39)

(d) n-butanol/n-propanol/0.1 N HCl, 2:2:1 (ascending) (109)

(e) n-butanol/acetic acid/water, 8:1:10 (ascending)

(f) dilsobutyl ketone/acetic acid/water, 40:30:7 (ascending) (67) 
The chromatograms were developed by spraying with a cresyl violet reagent ( 0.02 per cent in 1 per cent acetic acid) (67). Although the stained areas appeared streaked, better resolution was observed under ultra-violet illumination where the stained areas appeared dark blue on a red background. A variation in the mobilities of the two fractions was demonstrated in chromatograms irrigated in solvents (a), (b), (c), and (e). In solvent (c) three stained areas with $R_{f}$ values of $1.28,1.00$, and 0.63 were noted for Fraction \#1 of which the middle spot $\left(R_{f} 1.00\right)$ was the most intensely stained and the fastest spot $\left(R_{f} 1.28\right)$ wes the least intensely stained. Fraction $t 2$ wes also resolved into three areas with $R_{f} 0.98,0.61$, and 0.35 of which the middle spot $\left(R_{f} 0.61\right)$ was the major area. Stained areas corresponding to the areas denoted by $R_{f} 1.00,0.63$, and 0.35 were also observed with solvents (a), (b), and (e). Chromatography of increased quantities $(500 \mu \mathrm{g}$. ) of Fraction \#I in solvent (c) Indicated the presence of four stained areas. The chromatographic behavior of Fraction $\frac{\|}{\pi l}$ resembled that of van Heyningen's "fast" ganglioside preparation in solvent ( $f$ ), while Fraction $\frac{\| 2}{\| 2}$ resembled his "slow" ganglioside (67). Chromatography of both frections on borate impregnated paper (110) in several solvent systens resulted in severe streaking of the cresyl violet staining areas.

In an attempt to overcome the streaking and general ineffectiveness of paper chromatography for the distinct resolution of ganglitoside components, the two fractions were next subjected to TIC in a solvent consisting of chloroform/methanol/acetic acid, 3:6:1. Although Fraction \#1 was resolved into four cresyl violet 
staining areas, the resolution was not distinct. This result corresponded to the earlier observations with paper chromatography. Column II

Silicic acid with the properties already noted was slurried in chloroform and poured Into a clean glass column to produce a column of dimensions $42 \times 2.5 \mathrm{~cm}$. Ganglioside (950 mg.) with a NAM content of 31 per cent and 0.13 per cent phosphorus was placed on the column and covered with a filter paper disc. The column was filled with chloroform, and a gradient was established between one liter of chloroform/methanol, $5: 1$, in the mixing chamber and chloroform/ methanol, 1:1, in the reservoir. Tygon tubing was used for all connections. A magnetic stirrer on the mixing chamber insured mixing of the incoming solvent with the contents. Additional methanol/chloroform, 1:1, was added to the reservoir as required. Fractions of $15 \mathrm{ml}$. were collected on a fraction collector with a flow rate of $1 \mathrm{ml}$. per minute. The concentration of methanol in the eluate theoretically describes a convex curve asymptotically approaching chloroform/ methanol, $1: 1$ (111).

However, resorcinol-positive material was still being eluted from the column after three liters of eluate had been collected (Tube 225). The gradient elution was then continued from chloroform/methanol, 1:1, to methanol. This final segment of the gradient describes a second convex curve with methanol concentration in the eluate approaching abaolute methanol. Aliquots from each fraction were removed, dried under $\mathrm{N}_{2}$ in a warm water bath, and their contents of WNA and hexose were determined by resorcinol and anthrone assays, respectively. Elution of gangliostdes as 
evidenced by the resorcinol assay is shown in Figure 9. The fractions of maximal NANA concentrations are labeled. Anthrone analyses complemented the NANA analyses except for an anthrone peak in the area of fraction 95, which was resorcinol negative. This area was not investigated further. Allquots from fractions comprising the resorcinol peaks denoted in Figure 9 were removed for TIC in chloroform/methanol/acetic acid, 3:6:1. Five cresyl violet staining areas were observed. Although homogeneous $\rightarrow$ fractions were not obtained with this column, there did appear to be a separation of components. Of the five cresyl violet staining areas discernable by TIC, the two major components were seen in fractions 132 (Peak $B$ ) and 172 (Feak $C$ ), and the three less intense areas were noted in fraction 114 (Feak A) and in fractions following Peak C.

It was found that the resorcinol reagent (77) could be used as a definitive spray reagent for use with TIC.if, after spraying. the TIC plate was heated inside an HCI saturated, closed chamber. The purple color produced by the action of resorcinol in HCl is specific for sialic acid and sialic acid containing compounds. - Asialogangliosides produced by acid hydrolysis yielded brown spots with resorcinol on TLC which were readily distinguishable from the resorcinol-positive areas.

The effectiveness of this reagent as a spray for the distinctive resolution of gangliosides on TIC was even more pronounced with the introduction of the solvent system chloroform/methenol/water, 


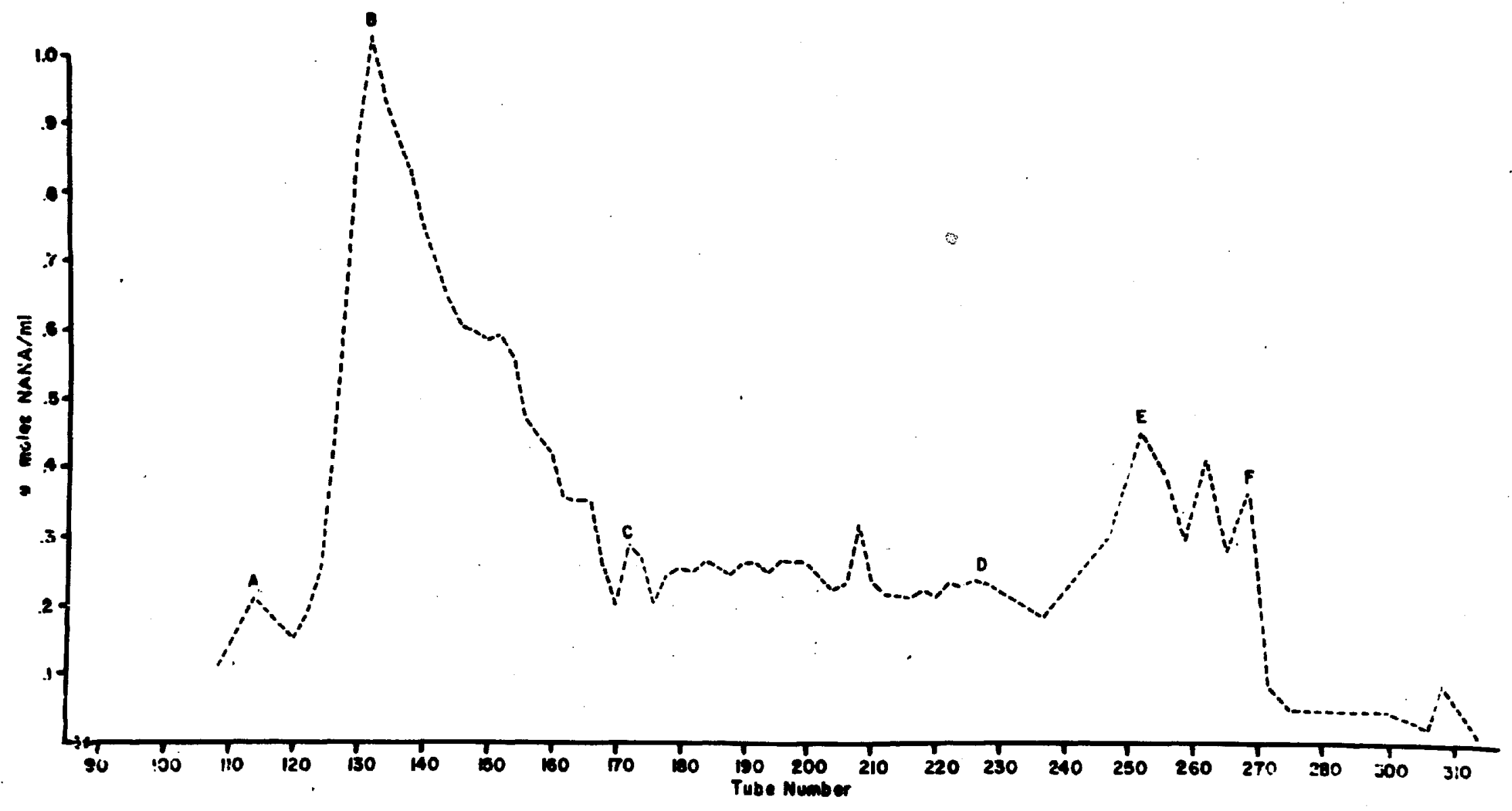

Figure 9.-Ganglioside Elution from Column II 
$60: 35: 8$ (93). These two techniques provide a very effective means for the distinct chromatographic resolution of gangliosides.

Four distinct resorcinol-positive spots were demonstrated with only minimal amounts of ganglioside (50-75 ug.) Figure 10 (R-right)). Three or four faster moving resorcinol-positive components could be observed when larger amounts were chromatographed; these components were faster moving than the four major gangliosides. These were observed on the original plate but were not reproduced in Figure 10 (R-left). They are, however, discernable in Figure 11 (Peak A). The nomenclature for the gangliosides in this thesis was developed from these results. The four major gangliosides of Figure 10 were designated in order of decreasing chromatographic mobility $1-G, 2-G, 3-G$, and 4-G. The faster moving minor gangliosides shown in Figure 11 were designated in the same order SG-1, SG-2, and SG-3. The component moving immediately ahead of the first major ganglioside, 1-G, designated FM can be seen in both illustrations (F'1gures 10 and 11).

Fractionation of the gangliosides on Column II resulted in a partial resolution of the different gangliosides. The fast moving gangliosides were eluted in Peak A (Figures 9,11). The major gangliosides were present throughout the elution gradient. 1-G was a component of Peak B (Figures 9,10), which also appears to contain 2-G. Peak D contained 3-G with very small amounts of the other gangliosides. GangIloside 4-G appeared concentrated in Peak F. Spotting of larger amounts of the fraction at the maximum of Peak $C$ (Tube 176) on another TIC plate showed clearly the presence of $3-G, 2-G$, and 1-G. The presence 


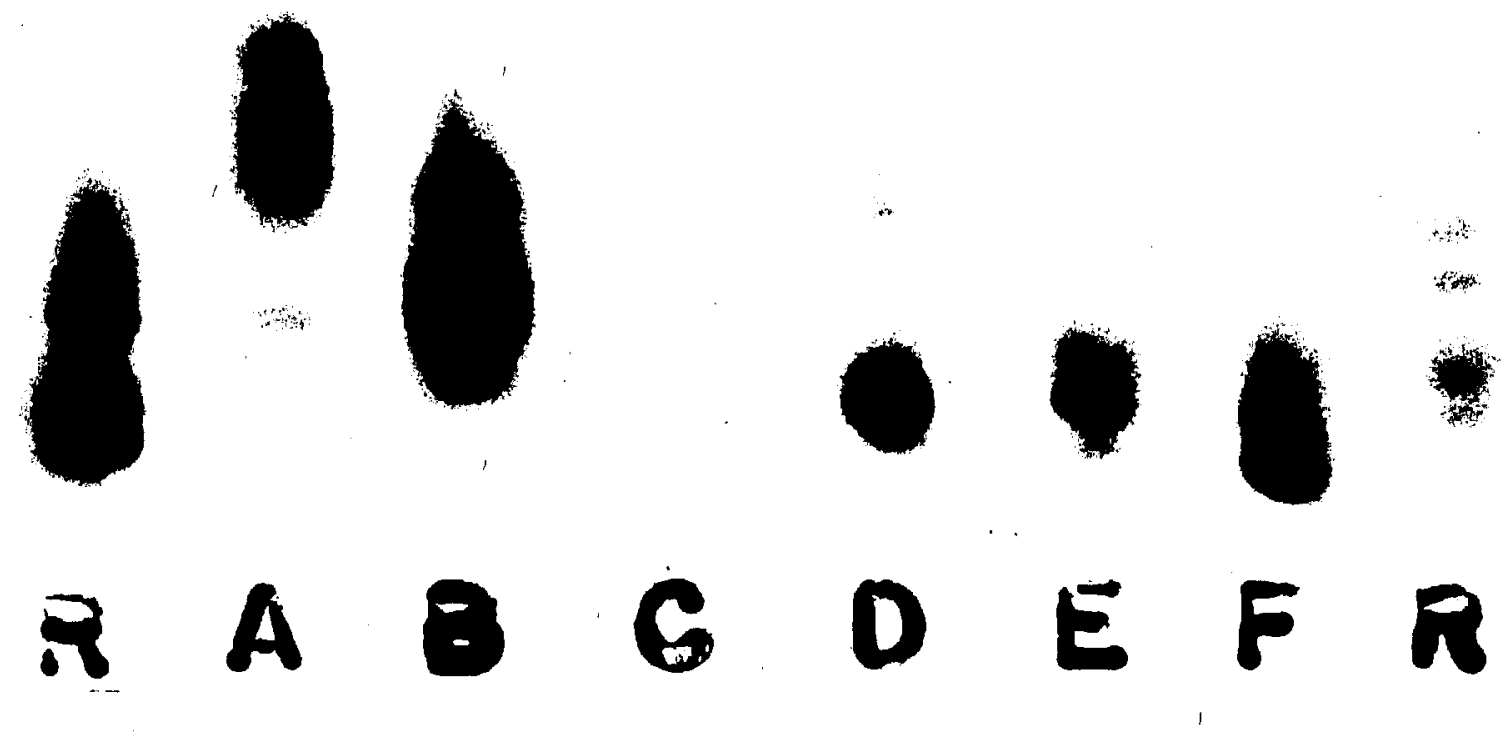

Figure 10.--Thin Layer Chromatography of Fractions from Column II. R-left) ganglioside preparation (700 $\mathrm{kg}$. ).

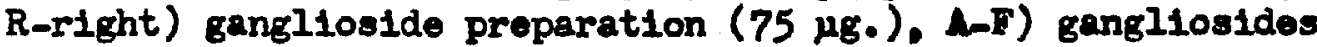
from correspondingly labeled peak tube fractions of Figure 9. Solvent: Chloroform/methanol/water, 60:35:8. Indicator: Resorcinol reagent and developed in a HCl saturated atmosphere. 


\section{SG-1 \\ $56-2$}
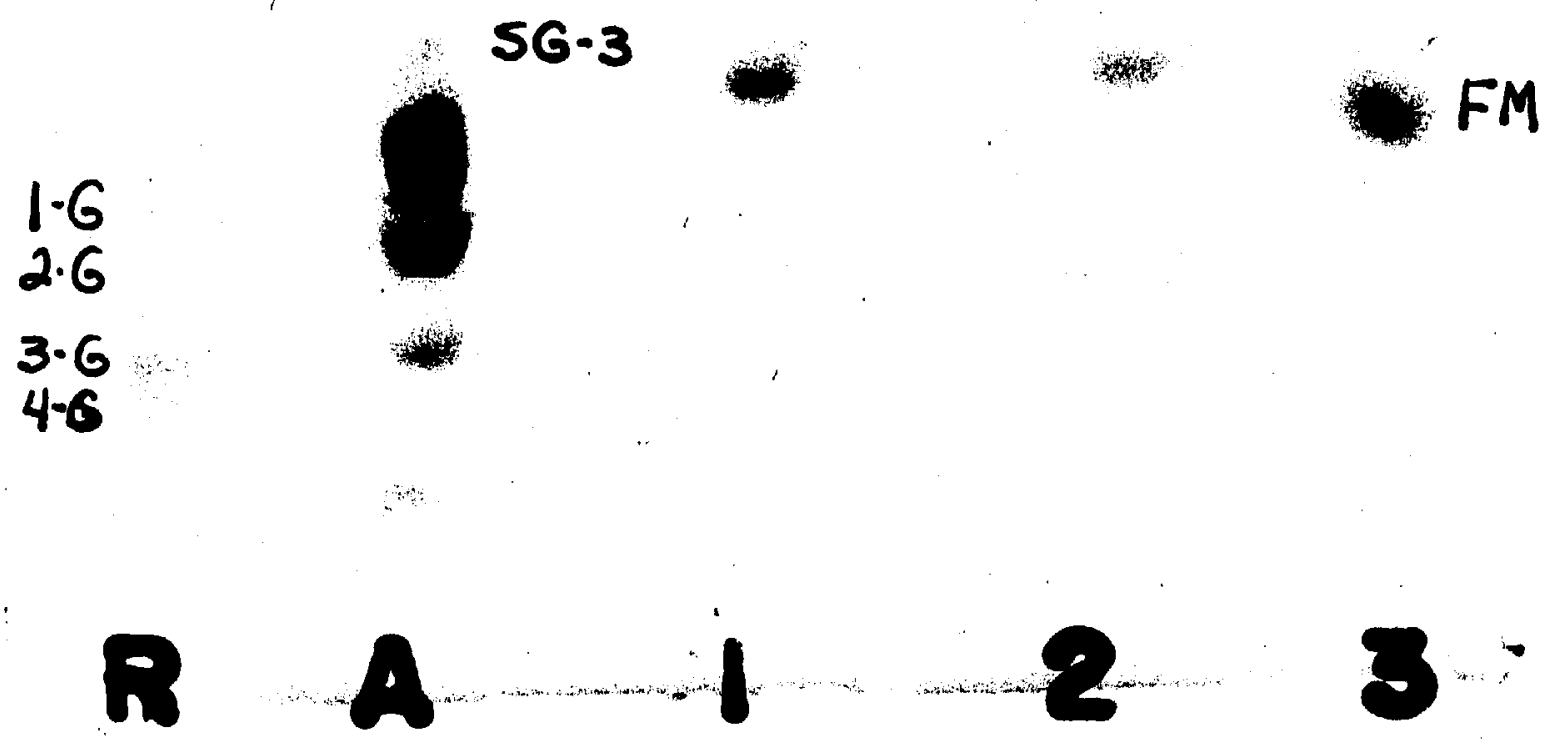

Figure 11.--Thin Layer Chromatography of Fast Moving. Minor Gangliosides. R) ganglioside preparation showing the four major gangliosides. A) gangliosides comprising Peak A of Column II (F1gure 9), 1,2,3) SG-1, SG-2, and FM, respectively, after isolation by preparative TLC procedure. Solvent: Chloroform/methanol/water, 60:35:8. Indicator: Resorcinol reagent and developed in a BCl saturated atmosphere. 
of a resorcinol-positive spot in Peak F (Figure 10) which was slower moving than 4-G can be discerned. This spot has not been characterized.

\section{Preparative Thin Layer Chromatography of Gangliosides}

In an attempt to isolate individual gangliosides, fractions comprising Peak (Figure 9) were pooled and the solvent concentrated by rotary evaporation. A yellow oil remained, a majority of which was thought to be the methanol/chloroform extract of the Tygon tubing used in Column II; it was removed with petroleum ether. The residue was dissolved in water and lyophilized. This material was dissolved in methanol/chloroform, spotted on TIC in $2-3 \mathrm{~cm}$. streaks and irrigated in chloroform/methanol/water, 60:35:8. A large number of plates with $500 \mu$ layers of silica Gel $G^{6}$ were required. The plates were air-dried, sprayed with the cresyl violet reagent, and corresponding stained bands from several plates were carefully scraped onto aluminum foil. This silicic acid was placed in a sintered glass filter where it was subjected to a thorough washing with chloroform/methanol and methanol until little or no color remained. The solvent was removed by rotary evaporation, the ganglioside and dye redissolved in water and dialyzed. Sodium hydroxide $(1 \mathrm{~N})$ was added to the dye-complex until the color of the solution changed to yellow ( $\mathrm{pH} 10.5$ ), the color extracted with cold ethyl ether, and the aqueous phase was passed over a Dowex 50N-X1 $\left(\mathrm{H}^{+}\right)$Ion exchange column. This eluate was dialyzed. The extraction process was done in the cold with as little delay 
as possible. The non-dialyzable material was lyophilized. This procedure will be referred to as preparative TIC.

Since the presence of the fastest moving gangliosides, SG-1 and SG-2, was detected only in Peak A (Figure 11), these gangliosides were isolated by the preparative TIC procedare. However, their chromatographic mobility was altered by this procedure in such a manner that both components now apparently had the same mobility (Figure 11-1 and 2). Sufficient amounts of both were not obtained to permit complete analysis. Solutions of the two were prepared, and aliquots were subjected to the resorcinol and anthrone analyses. Hexose to NANA ratio in SG-1 was 2.1 ; the ratio for SG-2 was 2.4 . Fractions 120-139 from Peak B were pooled and worked up as above for the fractions of Peak A (Figure 9). Three bands were observed when this material was spotted for preparative TLC and sprayed with cresyl violet. A small amount ( $8.7 \mathrm{mg}$. ) of the material comprising the fastest definite resorcinol-positive band was isolated. When rechromatographed in the same solvent (chloroform/methanol/water, 60:35:8), only one resorcinol-positive spot which corresponded to FM was observed (Figure 11-3). Compositional analyses of this material are shown in Table 6.

The second cresyl violet staining band which corresponded to 1-G, the fastest moving major ganglioside, was also isolated by this preparative TIC procedure. In contrast to the other materials this ganglioside (29 mg.) had a greenish color, and although it contained one principal component when rechromatographed, it was not homogeneous. Ion exchange chromatography of this ganglioside mixture 
on Dowex 50W-X1 $\left(\mathrm{H}^{+}\right)$did not remove the color. Single determinations by anthrone and resorcinol indicated a hexose to NANA ratio of 2.8 .

TABLE 6

ANALYSIS OF GANGLIOSIDE FM?

\begin{tabular}{lc}
\hline \hline & \multicolumn{1}{c}{} \\
NANA & 24 \\
Glucose & 9 \\
Galactose & 14 \\
N-Acetylhexosamine & 6 \\
Sphingosine & 17 \\
\hline & \\
\hline N-Acetylhexosamine and glucose \\
were analyzed in an I8 hour 2 \\
N HCl hydrolysate. Galactose \\
was determined as the differ- \\
ence between total anthrone \\
hexose and glucose determined \\
by the glucose oxidase method.
\end{tabular}

Chromatographic Eractionation of Gangliosides on Anasil S Columns

Gangliosides were chromatographed on small Anasil S columns using various solvents. The solvents employed in these preliminary runs were slight modifications of the solvent chloroform/methanol/ water. $60: 35: 8$. Conditions were found which indicated that chromatographically homogeneous gangliosides could be obtained. Three columns were employed in an attempt to isolate quantities in sufficient amounts for characterization.

9The analyses of this ganglioside were made in conjunction with Dr. K. Sambasivarao. 


\section{Column III}

A column ( $32 \times 1 \mathrm{~cm}$.$) was packed dry as indicated earlier with$ Anasil $S$ which had been activated by heating at $115^{\circ}$ for thirty minutes. Gangliosides ( $83 \mathrm{mg}$ ) which had been isolated according to the revised scheme (Figure 6) were mixed dry with additional Anasil and placed onto the column. The column packing was concluded with additional Anasil and a glass wool pad. An initial solvent of chloroform/methanol/water, 65:30:2, was employed with a flow rate of one-half ml. per hour. The polarity of this solvent was increased in order to $65: 30: 3,60: 35: 5$, and $60: 37: 8$. No resorcinol-positive material was eluted in the first three solvents. Aliquots were removed from each fraction and spotted on TIC plates for chromatography in chloroform/methanol/water, 60:35:8. Fractions containing only one resorcinol-positive spot on TLC were observed in the order of elution FM, 1-G, 3-G, and 4-G. Fractions containing only 2-G were not noted. However, the fast moving gangliosides were eluted together, and the degree of fractionation on this column was not as successful as the preliminary columns had demonstrated.

Column IV

A small column (11 × $1 \mathrm{~cm}$.) was packed dry as above with unactivated Anasil $S$ to which gangliosides (30 mg.) was applied dry. An initial solvent of chloroform/methanol/water, 65:30:2, was followed by chloroform/methanol/water, 60:35:5, and 60:37:8. Positive pressure was employed to establish a flow rate of $10 \mathrm{ml} / \mathrm{hour}$. Fractions of $1 \mathrm{ml}$. were collected manually from which aliquots were removed for TLC analysis. 
All four minor gangliosides were eluted as a mixture with the initial solvent. Fractions containing chromatographically homogeneous FM and fractions containing only 1-G were obtained. Additional amounts of homogeneous FM and 1-G were eluted with chloroform/methanol/water, 60:37:8. Fractions containing only 3-G and only 4-G were also obtained with this solvent. Again fractions containing only $2-G$ were not detected. Column V

Ganglioside (750 mg.) prepared from the revised ganglioside isolation scheme (Figure 7) and containing 28.4 per cent NANA and 0.13 per cent phosphorus was dissolved in chloroform/methanol to which a small amount of Anasil had been added. The excess solvent was evaporated under a stream of $\mathrm{N}_{2}$ in a warm water bath. The mixture was then placed onto the top of the column $(30 \times 3 \mathrm{~cm}$.). and the packing of the column was completed as in the previous two columns. One ml. fractions were collected manually with an injtial solvent of chloroform/methanol/water, 65:30:2. When gangliosides ceased to be eluted from the column as evidenced by TIC analysis, the solvent was changed to chloroform/methanol/water, $60: 37: 8$, and $4 \mathrm{ml}$. fractions were collected on a fraction collector. A flow rate of $16 \mathrm{ml}$./hour was maintained with the aid of positive pressure.

A faint yellow band of non-ganglioside material was first eluted from the column with the initial solvent. This band, which had been observed in the other Anasil columns, served as a marker, for it was closely followed by fractions that contained a mixture of four minor ganglioside spots. Fractions were then obtained 
which contained only FM. Fractions that contained a mixture of FM and 1-G were followed by several fractions containing only 1-G. Fractions which contained only a single resorcinol-positive area corresponding consecutively to FM, 1-G, 3-G, and 4-G were intermittently eluted with the second solvent. It was again noted that instead of $2-G$ following $1-G$ in the elution sequence, $a$ mixture of FM, 1-G and 2-G was eluted. Fractions which contained only 2-G were not detected.

A very slow moving resorcinol-positive material which did not correspond to any of the components in the original ganglioside preparation or to NANA was eluted last from the column with the second solvent.

Fractions were then pooled with special emphasis placed upon the retention of homogeneous characteristics of each ganglioside. Fractions from Column III and Column IV were combined when the homogeneity and identity of this material was assured by co-spotting, these fractions with fractions from Column $V$. After concentrating these combined fractions on the rotary evaporator, the solutions were lyophilized to yield white powdery residues for three of the four gangliosides. The lyophilization residue of FM had a slight yellow color with a more crystalline appearance.

Analyses of Chromatographically Homogeneous Gangliosides Removal of Silicic Acid from Gangliosides

Due to the presence of water in the eluting solvents and the very slight solubility of silicic acid in water, the four ganglioside preparations were expected to contain contaminating silicic acid. 
Removal of silicic acid from these gangliosides was necessary. An attempt was made to dissolve FM in a minimal volume of water. The material was only suspended and could not be filtered in a sintered glass filter until chloroform/methanol, approximately $1: 1$; was added. After lyophilization of the filtrate, a yellowish color still remained in the crystalline residue ( $23 \mathrm{mg}$. ). $1-G$ and 3-G were each placed in a sintered glass filter and $5 \mathrm{ml}$. of chloroform/methanol, approximately $1: 1$, followed by $3 \mathrm{ml}$. of acetone and then $5 \mathrm{ml}$. of water were used to wash the ganglioside through the filter. An additional $5 \mathrm{ml}$. of water was added to the filtrate, and the filtrate was lyophilized. White fluffy residues totaling $68 \mathrm{mg}$. and $85 \mathrm{mg}$., respectively, were obtained.

4-G, which did not appear to be soluble under these conditions in chloroform/methanol, approximately 1:1, was dissolved in water, filtered, and lyophilized. This residue ( $20 \mathrm{mg}$. ) resembled 1-G and $3-G$ in appearance.

Fractions containing the slow moving resorcinol-positive material which was last eluted from Column $V$ were pooled, concentrated by flash evaporation, and lyophilized. This material was not soluble in chloroform/methanol, approximately 1:1, and was rinsed from the lyophilization flask with water and stored in the refrigerator.

These four ganglioside preparations appeared homogeneous by TIC with chloroform/methanol/water, 60:35:8 (Figures 12,13), and with n-propanol/water, 7:3, when 50-75 $\mu$ g. were spotted. When larger quantities of FM were chromatographed, 1-G appeared as 
a contaminant (Figure 13). These same plates were oversprayed with $\mathrm{H}_{2} \mathrm{SO}_{4}$ /water, 1:1, and upon charring no spots other than the resorcinol staining areas were observed.

Characterization of $4-\underline{G}$

Carbohydrate composition of the lyophilization residue containing 4-G is indicated in Table 7.

\section{TABLE 7}

CARBOHYDRATE COMPOSITION OF LYOPHILIZATION RESIDUE CONTAINING 4-G

\begin{tabular}{lcccc}
\hline & Per Cent & Mmole/mg. & $\begin{array}{c}\text { Molar Ratio } \\
\text { Det'd }\end{array}$ & Assumed \\
\hline NANA & 27.6 & 0.89 & $1.0^{\mathrm{a}}$ & 3 \\
Hexosamine & 6.3 & 0.35 & 0.38 & 1 \\
Hexose & 17.9 & 0.99 & 1.07 & 3 \\
\hline
\end{tabular}

a Determined ratios are based on the NANA molar composition.

Although the percentage composition of this preparetion was not indicative of a trisialoganglioside, the molar ratio did suggest a trisialoganglioside. It was noticed that the chloroform/ methanol, 1:4, solution was slightly cloudy and contained suspended material which was not removed by filtration in a sintered glass filter. An attempt was then made to suspend the contaminating material in a solvent in which the ganglioside was not soluble. Acetone was added to the dried residue, swirled and decanted. The ganglioside was again dried under $\mathrm{N}_{2}$ to a constant weight, 


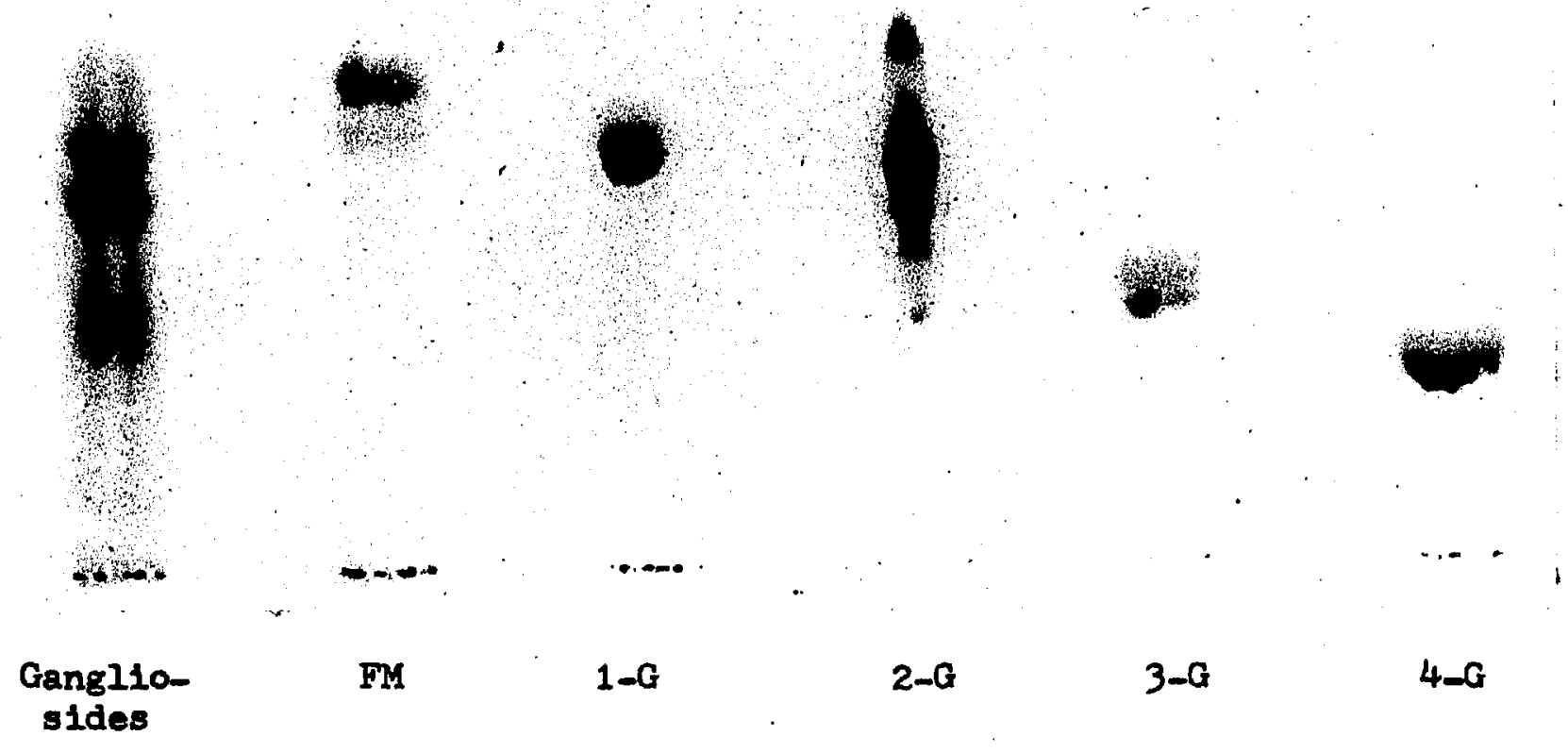

Figure 12.--Thin Layer Chromatography of Homogeneous Gangliosides (I). Material spotted consisted of a ganglioside preparation showing four major gangliosides, homogeneous preparations of gangliosides FM, 1-G, 3-G, and 4-G, and a heterogeneous preparm ation of 2-G. Solvont: Chloroform/methanol/water, 60:35:8. Indicator: Resoreinol reagent and developed in a HCl saturated atmosphore. 


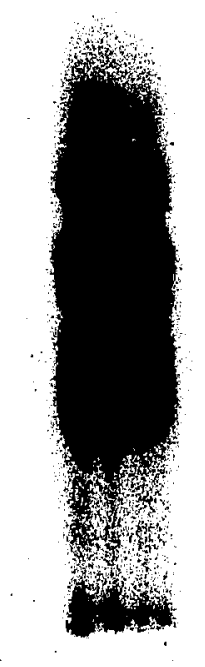

Gangliosides
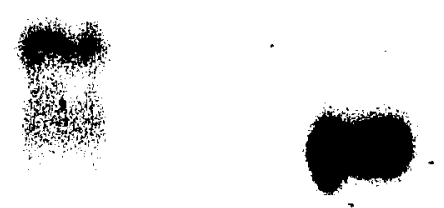

FM

$1-G$

$3-0$

4-G

Figure 13.-.Thin Layer Chrometography of Homogeneous Gangliosides (II). Material spotted consisted of a ganglioside preparation and preparations of FM, 1-G, 3-G, and 4-G. Solvent: Chloroform/methanol/water, 60:35:8. Indlcator: Resorcinol reagent and developed in a BCl saturated atmosphere. 
redissolved in chloroform/methanol, 1:4. This solution was now clear and apparently free of suspended material. An aliquot was removed for resorcinol analysis. The NANA percentage composition was 41 per cent. This procedure was repeated with additional material and the NANA content in duplicate analyses was 43.2 per cent, 41.7 per cent, and 42.9 per cent, which averaged 42.6 per cent. Analyses of the other carbohydrate components of this purified ganglioside 4-G are shown in Table 8.

\section{TABLE 8}

CARBOHYDRATE COMPOSITION OF 4-G

\begin{tabular}{lcccc}
\hline & Per Cent & Mmole/mg. & $\begin{array}{c}\text { Molar Ratio } \\
\text { Det'd Assumed }\end{array}$ \\
\hline NANA & 42.6 & 1.38 & $1.0^{2}$ & 3. \\
Hexosamine & 7.9 & 0.44 & 0.32 & 1 \\
Hexose $^{\mathrm{b}}$ & 24.5 & 1.36 & 0.99 & 3 \\
\hline
\end{tabular}

aetermined molar ratios are based on the NANA molar domposition.

$\mathrm{b}_{\mathrm{Galactose}}$ to glucose ratio was determined to be 1.7 .

\section{Characterization of $3-\underline{G}$}

Analyses of the carbohydrate composition of the ganglioside

3-G are shown in Table 9.

An attempt to increase the percentage composition of the carbohydrate components of this ganglioside using the acetone wash procedure was made with a small sample. NANA percentage content was found to be $31.1,29.6$, and 30.9 on three separate 
duplicate resoroinol analyses. However, upon repetition of thia wash procedure with larger quantities of $3-G$, a lower percentage composition was found. Hexose and hexosamine values on this preparation were comparably low. Nevertheless the molar ratios were 1.0 NANA, 0.51 hexosamine, and 1.4 hexose, assuming one mole of NANA.

TABLE 9

CARBOHYDRATE COMPOSITION OF 3-6

\begin{tabular}{|c|c|c|c|c|}
\hline & Per Cent & jmole/mg. & $\begin{array}{l}\text { Molar } \\
\text { Det'd }\end{array}$ & $\begin{array}{l}\text { Ratio } \\
\text { Assumed }\end{array}$ \\
\hline NANA & 28.8 & 0.97 & $1.0^{2}$ & 2 \\
\hline Hexosamine & 9.9 & 0.56 & 0.58 & 1 \\
\hline Hexoseb & 23.8 & 1.33 & 1.4 & 3 \\
\hline
\end{tabular}

\section{Characterization of 1-G}

Analyses of 1-G are illustrated in Table 10. Acetone washing of this ganglioside silghtly decreased the percentage composition as with 3-G, but the ratios were not altered. 
TABLE 10

CARBOHYDRATE COMPOSITION OF $1-G$

\begin{tabular}{lcccc}
\hline & Per Cent & $\mu$ moles/mg & $\begin{array}{c}\text { Molar Rat10 } \\
\text { Det'd }\end{array}$ & $\begin{array}{l}\text { Assumed } \\
\text { MANA }\end{array}$ \\
\hline Hexosamine & 19.9 & 0.63 & $1.0^{\mathrm{a}}$ & 1 \\
Hexose $^{b}$ & 12.0 & 0.67 & 1.07 & 1 \\
\hline
\end{tabular}

Determined ratios are based on the MNA molar composition. Galactose to glucose ratio was determined to be 1.9.

\section{Characterization of Fy}

The initial composition of the lyophilization residue containing FM was found to be 16 per cent NAira and 6.7 per cent hexosamine. The percentage composition of the carbohydrate units in the molecule was increased by washing with acetone, which also removed some of the color from the sample. Date resulting from analyses of this material are abown in Table 11.

\section{TABLE 11}

INITIAL CARBOHYDRATE COMPOSITION OF FM

\begin{tabular}{lcccc}
\hline & Per Cent & jumole/mg & $\begin{array}{c}\text { Molar Rat10 } \\
\text { Det'd }\end{array}$ & $\begin{array}{l}\text { Assumed } \\
\text { MANA }\end{array}$ \\
Hexosamine & 22.2 & 0.72 & $1.0^{\circ}$ & 2 \\
Hexose & 6.4 & 0.36 & 0.50 & 1 \\
& 20.5 & 1.1 & 1.6 & 3 \\
\hline
\end{tabular}

Datermined ratios aro besed on the Min molar composition. 
These ralues for IN based on the micromolar concentrations suggested a molooular composition consisting of 2 MANA, 1 hexosamine, and 3 hexose residues. However, the aqueous solubility characteristic of IM noted earlier together with its high mobility on TIC would not be predictod for a molecule of this composition. The diffieulty in dissolving this ganglloside in water suggested that increased acid strength might lead to more complete hydroln ysis. In an offort to determine the true composition of this fast moving ganglioside molecule, hydrolysis conditions of $3 \mathrm{~N}$ $\mathrm{H}_{2} \mathrm{SO}_{4}$ for five hours were employed for the hexose hydrolysis and $5 \mathrm{~N}$ HCl for seven hours for hexosamine. The values obtained by these hydrolysis conditions are shown in Table 12.

TABLE 12

CARBOHYDRATE COMPOSITION OF FM

\begin{tabular}{llccc}
\hline & Per Cont & jmole/mg. & $\begin{array}{c}\text { Molar Ratio } \\
\text { Dot'd Ascumed }\end{array}$ \\
\hline NANA & 22.2 & 0.72 & $1.0^{\mathrm{a}}$ & 1 \\
Hexosamine & $10.9^{\mathrm{b}}$ & 0.61 & 0.85 & 1 \\
Hexose $^{\mathrm{c}}$ & $25.0^{\mathrm{b}}$ & 1.4 & 1.9 & 2 \\
\hline
\end{tabular}

alolar ratios are based on the NANA molar composition. binimal values; for explanation see "Dlscussion". cGalactose to glucose ratio was determined to be 1.06 . 
Compartson of Composition for IY. 1-G, 2-G, and 4-G

A comparison of the carbohydrate composition of these four gangliosides is show in Table 13.

The presence of an aliphatic ceramide molety in each of the gangliosides is demonstrated by the strong C-H absorption bands at 3.4-3.5 $\mu$. The infrared spectra are shown in Figures 14-17. An infrared spectrum of the NANA standard used in this investigation is also included (Figure 18). 
TABLE 13

CARBOHYTRATE COAPOSITION OF FOUR HOMAN BRAIN GANGLIOSIDES

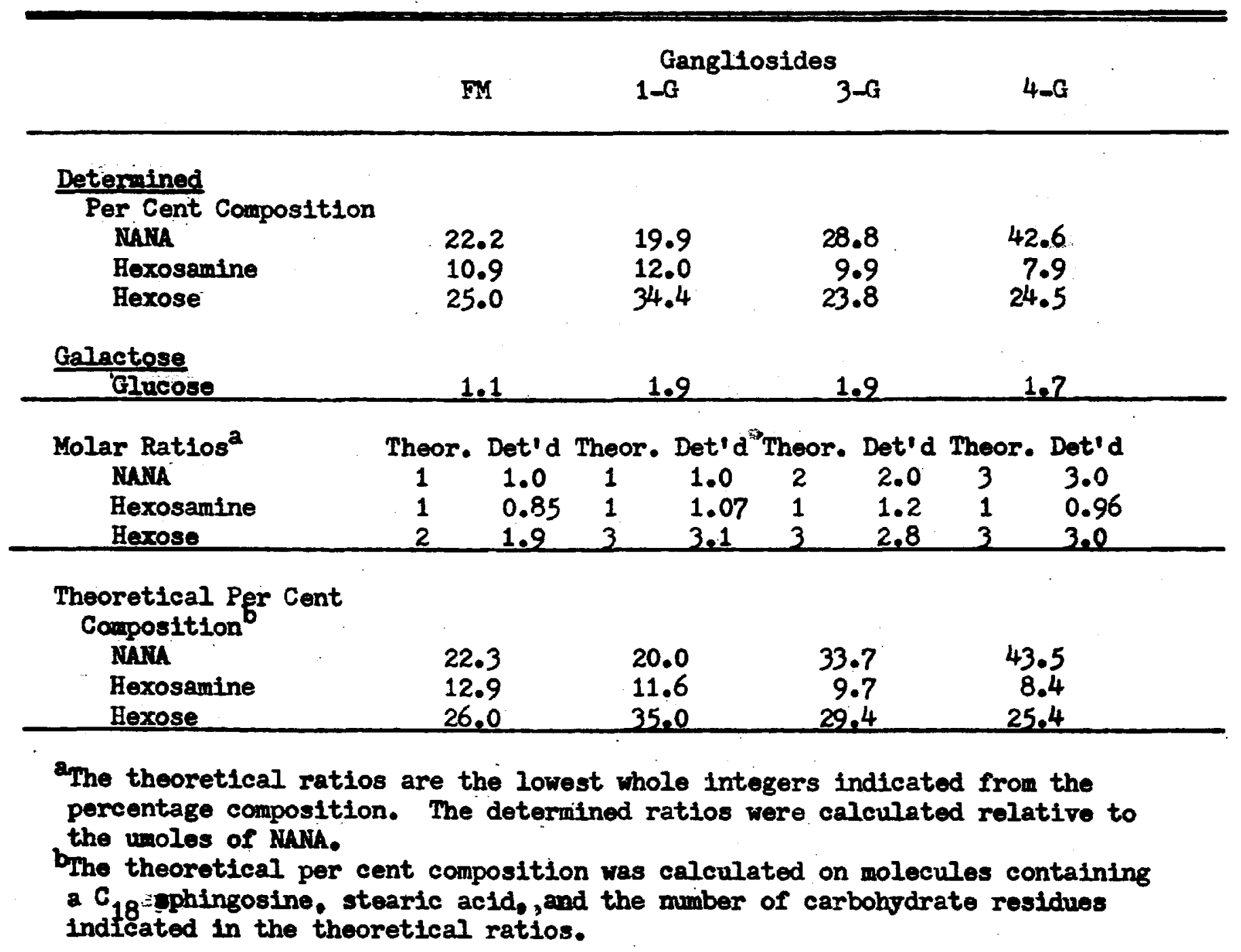




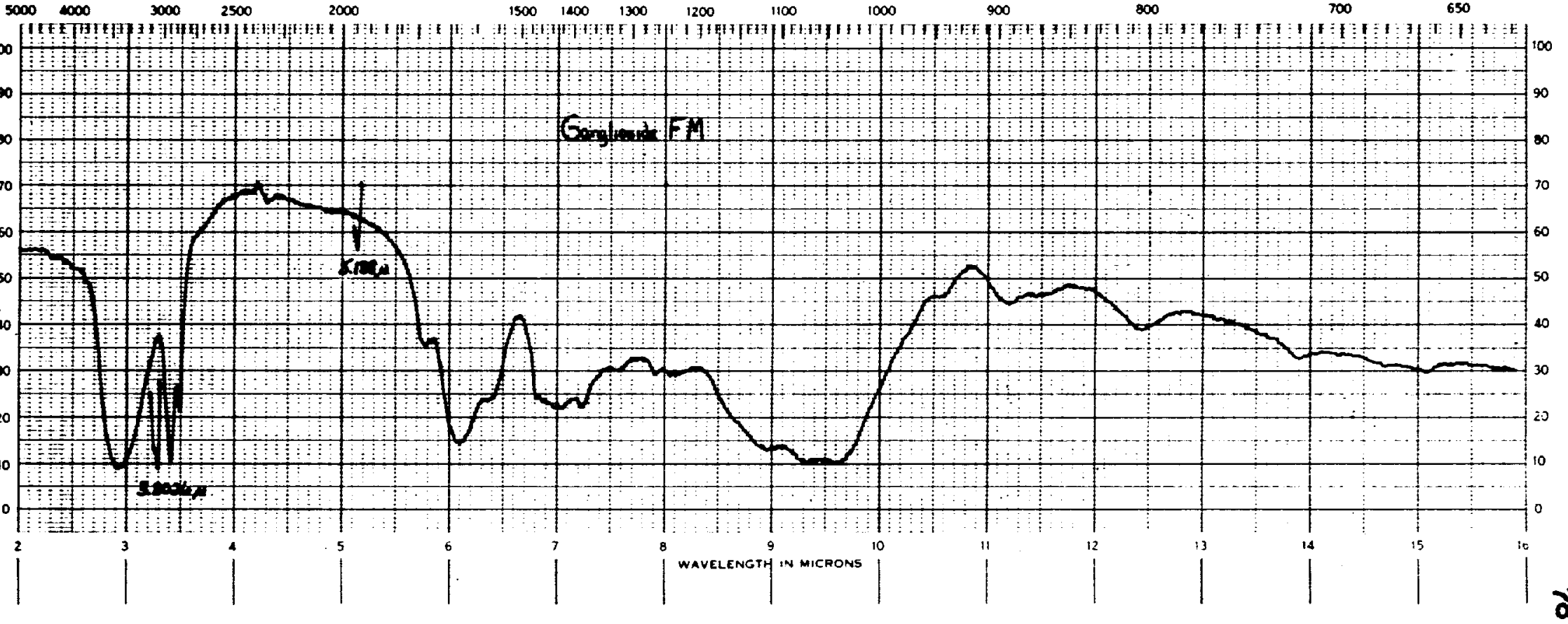




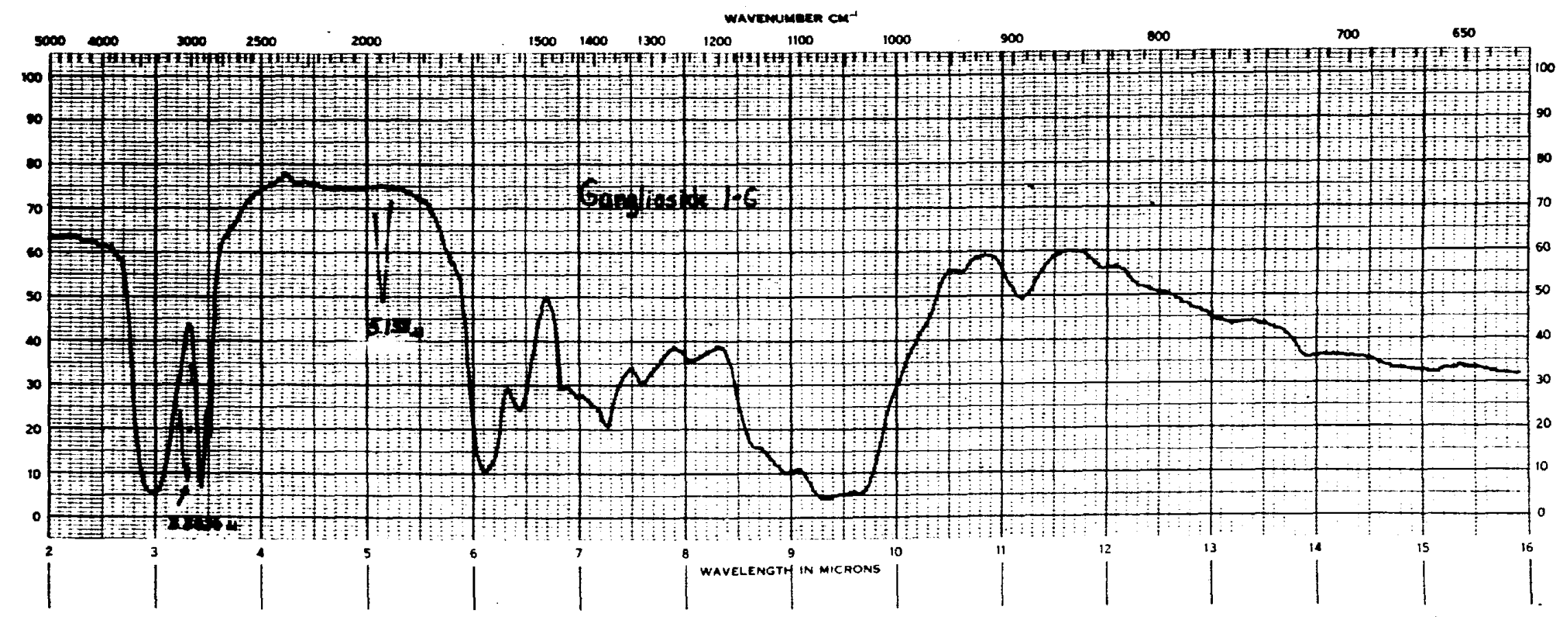

Figure 15.--Infrared Spectrum of Ganglioside 1-G 


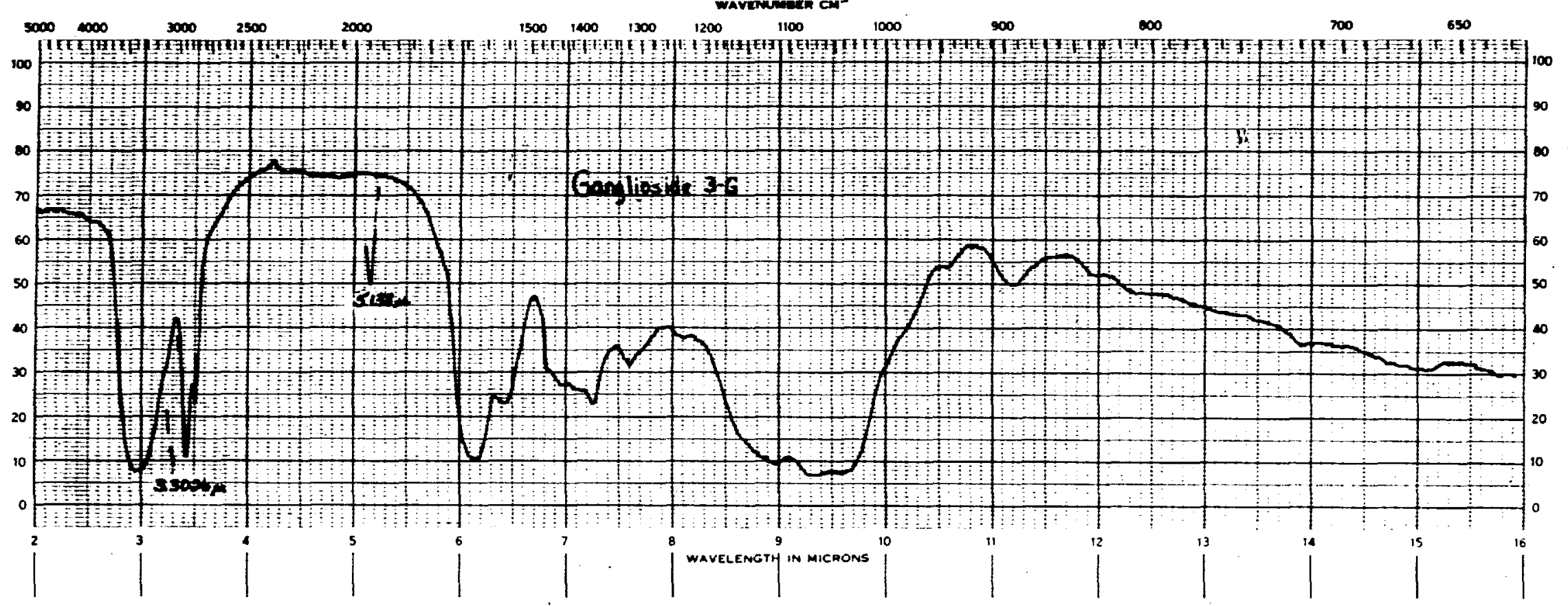

Figure 16.--Infrared Spectrum of Ganglioside 3-G 


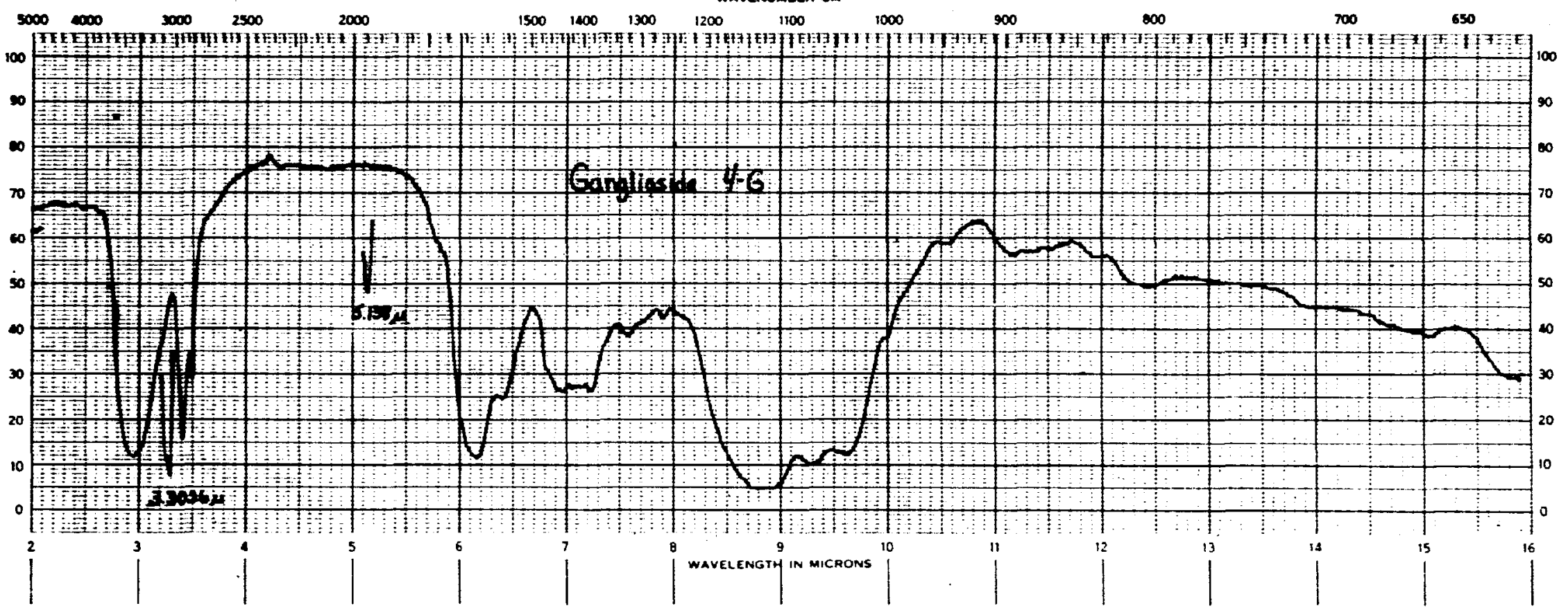

Figure 17.--Infrared Spectrum of Iyophilization Residue Containing Ganglioside 4-G 


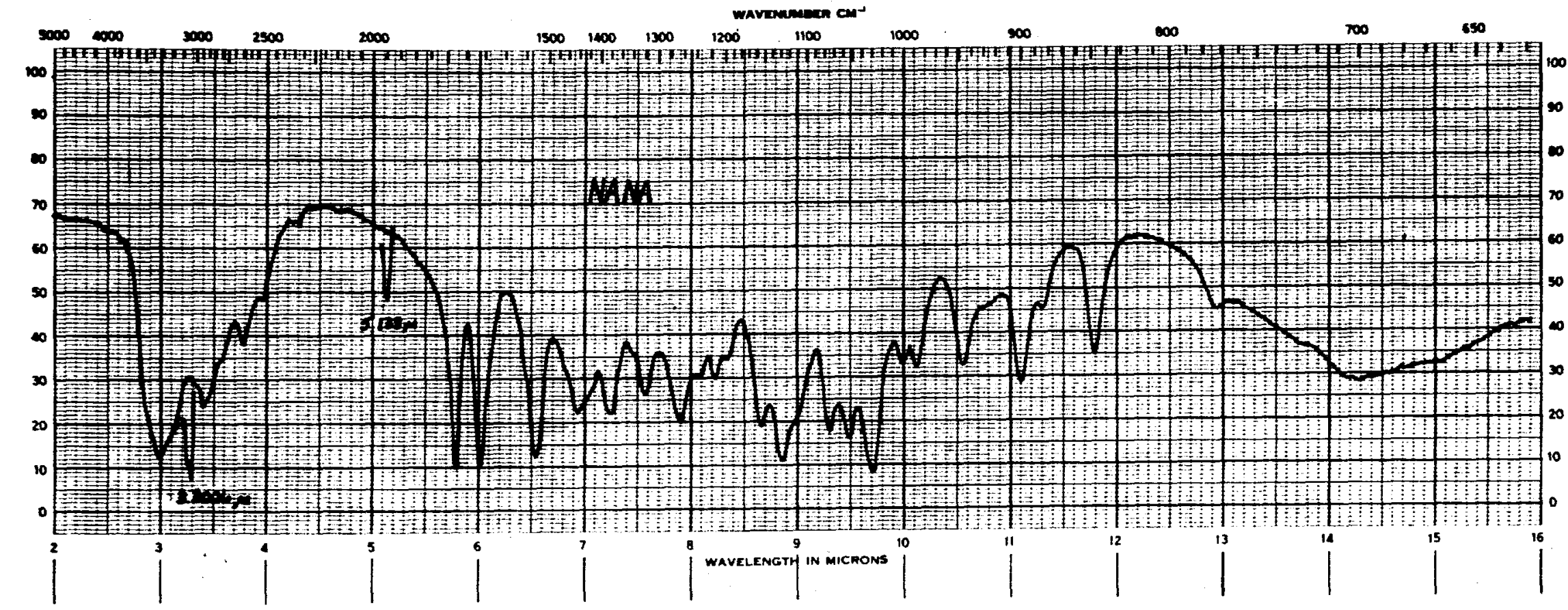

Figure 18.--Infrared Spectrum of N-AcetyIneuraminic Acid 


\section{DISCUSSION}

\section{Isolation of Gancliosides}

The methodology employed for the isolation of gangliosides In this thes10, while including techniques reported by others $(22,35,75)$. is sufficiently unique to warrant a thorough discussion.

Because the ganglioside content of human gray matter has been shown to be several times that of white matter (75). gray matter was used exclusively as the source of gangliosides in this investigation. Lyophilized gray matter was extracted with 95 per cent acetone to remove a large portion of the cholesterol and cerebrosides (75). Low solvent to tissue ratios and relatively short extraction periods were employed to prevent loss of gangliosides. Svennerholm (75) and McCluer et al. (81) have reported little or no loss of gangliosides in acetone extracts.

The residue of the acetone extraction was subjected to ether extraction in order to remove a portion of the phospholipids. Again a low solvent to tissue ratio and extraction in the cold were used to minimize the ganglioside loss. No resorcinol positive material was detected in these ether extracts. Svennerholm (75) has reported that considerable amounts of gangliosides are lost during Soxhlet extractions with ether.

In order to minimize the extraction of non-ganglioside-bound sialic acid, the acetone-ether dried residue was extracted with chloroform/methanol, 1:2. Svennerholn (75) has noted that 
chloroform/methanol extractions of wet tissue contain protein or peptide-bound siallc acid. This finding cast some doubt as to the exact nature of the ganglioside product obtained by the methods of Rosenberg and Chargaff (29), and Folch et 2l. $(22,35)$. Similar results may be expected with the hot methanol extraction of wet whole cerebral hemispheres as employed by Bogoch (26).

Chloroform/methanol extracts obtained in this investigation were then subjected to solvent distribution. The solvent system was that introduced by Folch et al. (35) in which gangliosides partition into the upper phase. The importance of the preliminary extractions with acetone and ether was clearly demonstrated at this step. The color in the chloroform/methanol extract was much less and the phase separation was much cleaner than in a similar extract prepared without the ether extraction step. This procedure in which each of the four lower phases was washed Individually and in sequence by the four upper phases, accomplishes a nearly complete separation of the gangliosides from the lipids of the lower phase (81). This distribution probably accomplished what extensive dialysis partitioning accomplished in the method of Bogoch (26) and in the method of Rosenberg and Chargaff (29).

Cation exchange chromatography (Dowex 50W-X1). which converted gangliosides into the free acid form, was originaliy employed in this method to remove amino acids and peptides which were not removed by dialysis. The nature of the materlal which remained at the top of the resin column after chromatography was not determined. The increase in the sialic acid content in the eluted gangliosides indicated the removal of non-ganglioside material. 
Reproducibility in the NAMA content (ca. 30 per cent), phosphorus content (0.15-0.25 per cent), and the chromatographic pattern on silicic acid thin layer of the ganglioside product isolated by this method has been noted.

\section{Chromatographic Tractionation of Gangliosides on} Silicic Acid Columns

Due to the suspected close structural and compositional relationships of the ganglioside molecules and to the successful separations of closely related lipids which had been achieved on silicic acid, silicic acid column chromatography was selected for the first attempt to fractionate gangliosides.

Selection of the initial solvent in Column I was made by noting the solubility of gangliosides in various chloroform/methanol mixtures. Chloroform/methanol, 3:1, was the least polar solvent to clearly solubilize gangliosides. The large resorcinol peak (Fraction \#1) and the gangliosides eluted after this peak (Fraction \#2) (Figure 8) showed different paper chromatographic behavior Indicating a partial fractionation of gangliosides had been achieved. The elution gradient employed in Column II afforded a very gradual change in the polarity of the eluting solvent. The concentration of the solvent leaving the mixing chamber as the anthrone peak was being eluted (Tube 95-Figure 9) was calculated by the nomograph method described by Warner and Lands (111) to be approx1mately chloroform/methanol, 3:2. The concentration of the solvent leaving the mixing chamber at the maximum of Peak B (Tube 132Figure 9) was similarly calculated to be chloroform/methanol, 
1.38:1. This polarity was slightly greater than that of chloroform/ methanol, 3:1, with which the large resorcinol peak in Colum I was eluted. The anthrone-positive peak preceding the elution of resorcinol-positive materlal was most likely caused by the contamination of less polar glycoliplds in this ganglioside preparation. In spite of the gradient elution, this column was not successful for the resolution of individual gangliosides.

\section{Preparative Thin Leyer Chromatography of Gangliosides}

Small amounts of FM and I-G were isolated by the preparative TIC procedure. SG-1 and SG-2 were also 1solated, but their mobility was altered during the procedure (Figure 11-1,2). The inforence 1s that both gangliosides contain an alkall labile group or linkage which when broken would make the molecule more polar, 1.e.. less mobile on TIC." However, the acidity of the ion exchange colum may also have affected these two gangllosides. Numerous plates were required for the isolation by this method, and the recovery of ganglioside from the TIC plates approximated 60 per cent. Attempts to isolate 2-G yielded a greenish residue, which upon chromatographing resulted in four resorcinolpositive areas. The color in this preparation and in the 1-G which had been similarly isolated was thought to be due to a trace of copper that was introduced to silicic acid during its sereening through copper alloy containing sieves (112).

The applicability of this TIC preparative procedure for the isolation of gangliosides does not generally compare favorably to the Anasil s column fractionation. However, until a fractionation 
scheme is developed for the isolation of the minor ganglioside SG-3. this procedure may find utility.

Chronatomesphte Fractionation of Gangliosides on Anini S Colume

Fractionation of gangliosides on Anasil $S$ columns resulted in the elution of homogeneous preparations of four gangliosides. FM. 1-G, 3-G, and 4-G. Resolution of the three fastest moving gangliosides, SG-1, SG-2, and SG-3, was not effected, and homogeneous 2-G was not obtained. Modification of the column packing. dimensions, and solvent systems may lead to resolution of each of the gangliosides. Sufficlent amounts were obtained by this method to permit the characterization of the gangliosides thus resolved.

\section{Removal of Stlicic Acid from Gangliosides}

The elution of column packing material in column chronatographic procedures has been noted $(39,54,90)$. Material present in the lyophilization residue containing 4-G had not been removed by filtering either in an aqueous solution or in a chloroform/methanol, 1:4. Since Svennerholm had employed acetone to precipitate gangliosldes from a cellulose column eluate and to suspend the cellulose, acetone was used here in an attempt to remove silicic acid present In this 4-G containing residue. The results of this washing procedure can be observed in the increase of the percentage composition values of 4-G in Table 8 over the values of. Table 7. Similar results were noted in FM (Table 11).

Although the molar ratios of the carbohydrate components in 1-G. and 3-G approximated more closely their assumed ratios after 
washing with acetone (Table 9 and Table 10), a decrease in percentage composition for these components was noted. This decrease was most likely due to a weighing error.

\section{Analrses of Chromatosraphically Homogeneous Gangliosides}

Hexose values for the isolated gangliosides increased slightly after mploying $2 \mathrm{~N} \mathrm{H}_{2} \mathrm{SO}_{4}$ for the hydrolysis of gangliosides. Hydrolysis conditions for the determination of hexosamine were not altered for 1-G, 3-G, and 4-G after the time course study had been completed.

The increased percentage composition of hexose and hexosamine in FM resulting from decreased hydrolysis time and increased acid strength indicated that the initial hydrolysis conditions were one or a combination of: (1) too short hydrolysis time, (2) too long hydrolysis time, (3) a solubility problem. The observation concerning the solubility characteristic of $\mathrm{FM}$ in water prompted the change to hydrolysis with stronger acid. However, these more vigorous hydrolysis conditions may not have yielded complete hydrolysis of FM, and the values reported for hexose and hexosamine composition should be regarded as "minimal values".

Hydrolysis of the hexosamine standard, N-acetylglucosamine, under these more vigorous conditions resulted in a slightly higher optical density in the hexosamine analysis than had been repeatedly observed for an equel amount of standard subjected to the hydrolysis conditions employed for the other gangliosides. This result indicated that the hexosamine values for $1-G, 3-G$, and $4-G$ might bo 
as much as five per cent high. The slightly lower value for the 18 hour hexosamine standard hydrolysate was probably due to degradation.

The sphingosine assay of Lauter and Trams (104) requires several mg. of ganglioside for a determination and, by the authors' own admission, gives low results (37)." since the molar ratios of the component carbohydrate residues in each of the gangliosides were Indicative of their composition, evidence indicating the presence of the aliphatic ceramide molety would be sufficient to prove that these products were indeed gangliosides. This evidence was obtained by infrared spectrophotometric analyses of the isolated gangliosides. Each of the gangliosides exhibited strong C-H absorption bands at 3.4 and $3.5 \mu$. Very similar absorption bands were shown by Makita and Yamakawa (113) for cerebroside and ceramide-dihexosides.

\section{Pature of Fuman Gangliosides}

Human ganglioside preparations were resolved.with the aid of column chromatography and TIC into eight resorcinol-positive components. Four of these gangliosides were present in major amounts, while four minor occurring gangliosides were also detected. The three fastest moving gangliosides, SG-1, SG-2, and SG-3, were the least abundant, and each appeared to contain a hexose:itill ratio of two. The presence of eight chromatographically resolvable resorcinol-positive areas on TIC has only recently been noted by others (115). Whether these minor gangliosides are natural or are created during isolation would be difficult to determine. 
FM, the slowest moving of the minor occurring gangliosides. contains galactose, glucose, hexosamine, and NAir in equimolar ratios. This ganglioside corresponds in composition to "Ganglioside $A^{n}$ of Klenk and Geilin (83), and to the "Tay-Sachs' ganglioside" of Svennerholm (95). As each of the minor gangliosides contains what appears to be a hexose to NANA ratio of two, which is the same as that reported for the ganglioside in Tay-Sachs' disease, positive identification of this ganglioside must awat the isolation of gangliosides from a Tay-Sachs' brain. The amount of FM isolated. large in comparison to the one per cent of the Tay-Sachs' ganglioside noted by Svennerholm (95), may be too large to compare favorably. However, possible molecular interconversions of $2-G$ to $1-G$ and $F M$ are suggested by the chromatographic elution behavior.

1-G is a monosialoganglioside containing galactose, glucose, hexosamine, and NANA in the ratio $2: 1: 1: 1$. This ganglioside is identical in composition and in relative chromatographic behavior to the monosialoganglioside of Svennerholm (95) and $G_{1}$ of Kuhn et a‥ (41).

Disialoganglioside 3-G corresponds in composition to the two disialogangliosides noted by Kuhn et al. (41) and the three resolved on TIC by Svennerholm (95). 3-G contains galactose, glucose, hexosamine, and MANA in the ratio $2: 1: 1: 2$." Chronatographical1y 3-G corresponds to $G_{3}$ of Kuhn and disialoganglioside $b$ of Svennerholn. The only previous report noting the occurrence of a ganglioatde molecule containing three sialic acid residues was that $\left(G_{4}\right)$ bo Kuhn et a]. (41). Trisialoganglioside 4-G contains galactose. 
glucose, hoxosamine, and WNA in the ratio $2: 1: 1: 3$. The composition of the four major gangliosides, according to svennerholm (95). consists of a mono- and three dislalogangliosides $(a, b, c)$. The chromatographic behavior of dinlaloganglioside c resembles that of $4-G$ in the same solvent system.

Although a chromatographically homogeneous preparation of 2-G was not isolated, the relative chromatographic mobility of this component suggests that $2-G$ is the second disialoganglioside $\left(G_{2}\right)$ described by Kuhn et all. (41).

The carbohydrate composition of gangliosides reported by various investigators is shown in Table 14.

Gangliosides 1-G and 3-G have been shown by Drs. M. C. Dodd and N. J. Bigley, Department of Microblology, to inhibit the agglutination of $\mathrm{Rh}_{\mathrm{O}}(\mathrm{D})$ antigen with $\mathrm{Rh}$ (D) antibody. A maximum $4+$ agglutinating system was completely inhibited by one ug. of ganglioside. The lyophlilzation residue containting 4-G (Table 7) also inhibited this system but much less effectively. 
TABLE 14

COMPARISON OF GANGLIOSTDES REPORTED

BI VARIOUS INVESTIGATORS

\begin{tabular}{|c|c|c|c|c|c|c|}
\hline \multirow[b]{2}{*}{ Reference } & \multirow[b]{2}{*}{ No. } & \multirow[b]{2}{*}{ Ganglioside } & \multicolumn{4}{|c|}{ Composition } \\
\hline & & & Glu & $\mathrm{Gal}$ & $\mathrm{Ga}_{2} \mathrm{NH}_{2}$ & NANA \\
\hline $\begin{array}{l}\text { Klenk \& Geilin } \\
\quad(54,83)\end{array}$ & 4 & $\begin{array}{l}A \\
B \\
C \\
D\end{array}$ & $\begin{array}{l}1 \\
- \\
-\end{array}$ & $\begin{array}{l}1 \\
- \\
-\end{array}$ & $\begin{array}{l}1 \\
1 \\
0 \\
0\end{array}$ & $\begin{array}{l}1 \\
- \\
- \\
-\end{array}$ \\
\hline Kuhn et aㅡ. (41) & 4 & $\begin{array}{l}G_{1} \\
G_{2} \\
G_{3} \\
G_{4}\end{array}$ & $\begin{array}{l}1 \\
1 \\
1 \\
1\end{array}$ & $\begin{array}{l}2 \\
2 \\
2 \\
2\end{array}$ & $\begin{array}{l}1 \\
1 \\
1 \\
1\end{array}$ & $\begin{array}{r}1 \\
2 \\
2 \\
3\end{array}$ \\
\hline Svennerholm (95) & 5 & $\begin{array}{l}\text { Tay-Sachs' } \\
\text { Monosialo- } \\
\text { Disialo- a } \\
\text { Disialo- b } \\
\text { Disialo- c }\end{array}$ & $\begin{array}{l}1 \\
1 \\
1 \\
1 \\
1\end{array}$ & $\begin{array}{l}1 \\
2 \\
2 \\
2 \\
2\end{array}$ & $\begin{array}{l}1 \\
1 \\
1 \\
1 \\
1\end{array}$ & $\begin{array}{l}1 \\
1 \\
2 \\
2 \\
2\end{array}$ \\
\hline This study & 8 & $\left.\begin{array}{c}S G-1 \\
S G-2 \\
S G-3\end{array}\right\}$ & $\begin{array}{l}2 \\
1 \\
1 \\
\overline{1} \\
1\end{array}$ & $\begin{array}{l}\text { Hexose } \\
\qquad \begin{array}{c}1 \\
2 \\
\overline{2} \\
2\end{array}\end{array}$ & $\begin{array}{l}- \\
1 \\
1 \\
-1 \\
1\end{array}$ & $\begin{array}{l}1 \\
1 \\
1 \\
\overline{2} \\
3\end{array}$ \\
\hline
\end{tabular}

${ }^{\text {a }}$ SG-1 and SG-2 were isolated, but their mobllities were altered to that of SG-3. Identity of hexose residues was not determined. 


\section{SUMMARY}

Gangliosides were isolated from dried human gray matter employing a chloroform/methanol, 1:2, extraction of acetone and ether dried residue. After removal of the solvent, the extracted residue was subjected to a 4-tube double-withdrawal distribution using Folch solvents. The combined upper layers were concentrated, dialyzed, and passed through a Dowex 50W-X1 (H+) resin column. The column eluate was lyophilized yielding a white powder.

An attempt to fractionate the ganglioside preparation was made using silicic acid column chromatography and chloroform/ methanol solvent mixtures of increasing polarity. Paper chromatographic behavior of the two resulting fractions indicated that a partial fractionation had occurred.

Fractionation of gangliosides on a second silicic. acid column with an elution gradient extending from chloroform to methariol was also partially successful. Thin layer chromatography (TIC) of various fractions of the gangliosides eluted showed eight resorcinolpositive spots. Four of the spots corresponded to areas observed when only a minimal amount of the unfractionated ganglioside preparation was subjected to TIC. These major occurring gangliosides were, designated 1-G, 2-G, 3-G, and $4-G$ in order of decreasing chromatographic mobility. Four minor, fast moving gangliosides were concentrated in the early fractions and were 
1dentified in the same order SG-1, SG-2, SG-3, and MM." Attempts to 1solate SG-1 and SG-2 by a preparative TIC procedure resulted in a change in mobility of both gangliosides to that of sG-3. Preparation's of FM and 1-G were 1solated by this procedure.

A successful fractionation of a ganglioside preparation into homogeneous gangliosides was achieved on an Anasil $S$ dry-packed column. Fractions containing the four minor gangliosides were eluted with chloroform/methanol/water, 65:30:2; fractions containing only FM and 1-G were also obtained. Homogeneous FM, 1-G, 3-G, and 4-G were consecutively eluted with the second solvent chloroform/ methanol/water, 60:37:8. Fractions containing only 2-G were not obtained. Sufficient, amounts of chromatographically homogeneous gangliosides were obtained to permit further characterization.

Ganglioside FM was found to contain equimolar quantities of galactose, glucose, hexosamine, and MÄN.

The monosialoganglioside 1-G contained galactose; glucose, hexosamine, and NANA in the ratio $2: 1: 1: 1$.

3-G was found to be a disialoganglioside containing galactose, glucose, hexosamine, and NANA in the ratio $2: 1: 1: 2$.

NANA composition of $4-G$ was determined to be 42.6 per cent. This ganglioside was found to be a trisialoganglioside containing galactose, glucose, hexosamine, and NaiNA in the ratio $2: 1: 1: 3$.

The presence of the aliphatic ceramide molety in each of these gangliosides was demonstrated by infrared analyses.

Thus three of the four major gangliosides were 1solated, and their carbohydrate composition was determined. These were shown 
to be a mono-, a di-, and a trisialoganglioside. One of the minor gangliosides, FM, was also 1solated and analyzed. Its carbohydrate composition is identical with that reported for the major ganglioside of Tay-Sachs' disease. 


\section{BIBLIOCRAPHY}

1. Kenk, E., Hoppo-Seyler's Z, phyeiol. Chem.., 235, 24(1935).

2. B11x, G., Skand. Arch. Phys101., 80, 46(1938).

3. Klenk, E., Hoppe-Seyler's Z. physiol. Chem., 273, 76(1942).

4. Walz, E., Hoppe-Seyler's Z, physiol. Chem..166, 210(1927).

5. B1al, M., Deut. med. Wochschr., 28. 253(1902).

6. Levene, P. A., and K, Landsteiner, J. B10l. Chem.. 25, 607(1927).

7. Klenk, E., Hoppe-Seyler's Z. phystol. Chem., 262, 128(1939).

8. Klenk, E., Hoppe-Seyler's Z. physiol. Chem., 267, 128(1940).

9. Klonk, E., and H. Langerboins, Hoppe-Seyler's z. physiol. Chem.. 270. $185(1941)$.

10. Kenk, E., Hoppe-Seyler's Z, physiol. Chem., 268, 50(1941).

11. BIIx, G. I. Svennerholm, and I. Werner, Acta Chem. Scand. 4. $717(1950)$.

12. Blix, G., and L. Svennerholm, Acta Chem. Scand., 6. 358(1952).

13. Deuel, H. J.. in The Ilolds, volume 1, Interscience Fublishers, Inc. . 1951. p. 477 .

14. Gottschalk, A. The Chemlstry and Blology of Slal1c Acide and Related Substances, Cambridge University Press, 1960.

15. Renk, F., and F. Rennkamp, Hoppe-Seyler's Z. phys101. Chem.. 222. $253(1942)$.

16. Schuwirth, K., Hoppe-Seyler's Z. physiol, Chem., 278, 1(1943).

17. Yamakawa, T., and S. Suzuk1, J. Blochem. (Tokgo), 38, 199(1951).

18. Tamakawa, T., K. Yatsumato, and S. Suzuk1, J. B1ochen. (Tokyo), 43. $63(1956)$.

19. Nonk, E., and $\mathrm{K}$. Heuer, Deut. Z. Vordauungs- u. Storfwechealkrankh., 20, 180(1960). 
20. Kuhn, R, H. Egge, R. Brossmer, A. Gauhe, P. Klesse, W. Lochinger, E. Rohm, H. Trischmann, and D. Tschampel, Angew. Chem.. 22, $805(1960)$.

21. Kuhn, R., and R. Brossmer, Chem. Ber., 24, 252(1962).

22. Folch, J., S. Arsove, and J. A. Meath, J, Biol. Chem., 191. $819(1951)$.

23. Chatagnon, C.. and P. Chatagnon, Bull. soc. chim. biol.. 36. $373(1954)$.

24. Rosenberg, A., C. Howe, and E. Chargaff, Nature, 127, 234 (1956).

25. Svennerholm, I., Acta Chem. Scand.. 10, 694(1956).

26. Bogoch, S. Biochem. J., 68, 319(1958).

27. Harr1s, A. F., and A. Saffer, in Cerebral Sphingoltpldoses:

1 srmposiun on Tar-Sachs Disease and NIIled Disorders. Academic Press, 1962, p. 271.

28. Rosenberg, A., and E. Chargaff, Biochim. et Biophys. Acte, 21. $588(1956)$.

29. Rosenberg, A, and E. Chargaff, J, Biol, Chem, 232, 1031(1958).

30. Klenk, E., and H. Debuch, Ann. Rev. Biochem.. 28, 39(1959).

31. Hakomori, S., and R. Jeanloz, J. Biol. Chem., 236, 2827(1961).

32. Yamakawa, T., M. Matsumoto, S. Suzuki, and T. I1da, J. Blochem. (Tolyo), 43. 301(1956).

33. Uzman, I. I., Arch. Path., 55, 181(1953).

34. Berman, E. R., and S. Gatt, in Cerebral Sphingolipidoses: A Sxmposinu on Tar-Sachs' Disease and Allied Disorders, hcademic Press, 1962, P. 238.

35. Folch, J., M. Lees, and G. H. S. Sloane-Stanley, J. Blol. Chem., 226. $497(1957)$.

36. Svennerholm, L., Nature, $177,524(1956) ; 1,42(1956)$.

37. Trams, E. G. and C. J. Lauter, Blochim, et Blophys. Acts, 60. $350(1962)$.

38. Payne, S. H., and B. S. Platt, J. Neurochem., 2. 5(1961).

39. Rouser, G., A. J. Baunann, G. Kritchersky, D. Heller, and J. S. O'Brien, J. An. O1I Chemists' Soc.. 38, 544(1961). 
40. Klenk, E., and W. Gellin, Hoppe-Seyler's Z. physiol. Chem., 319. $283(1960)$.

41. Kuhn, R., H. Egge, and H. Wiegandt, Angew. Chem., 73, 580(1961).

42. Tanford, C.., in Symposium on Protesn Structure, ed. A. Neuberger, John W1ley and Sons, Inc., 1958, p. 39.

43. Harris, A, F., and F. Saifer, J. Neurochem., 5, 383(1960).

44. Harris, A. F., F. Saifer, and S. K. Weintraub, Arch. Biochem. Blophys.. 25. 106(1961).

45. Albers, R. W., and G. J. Koval, Biochim. et Biophys. Acta, 60, $359(1962)$.

46. Gatt, S., Bull, Res. Counc1l Israel, 11A, 90(1962).

47. Harris, A. F., and F. Saifer, J. Neurochem., 5, 218(1960).

48. Rapport, M. M., and W. T. Norton, Ann. Rev. Biochem., 31. $128(1962)$.

49. Mandelstam, P., and R. M. Burton, Fed. Proc., 18, 280(1959).

50. Moser, H. W., and M. L. Karnovsky, J. Biol. Chem., 234, 1990(1959).

51. Brady, R. 0., J. Biol. Chem., 237, 2416(1962).

52. Kishimoto, Y., and N. S. Radin, J. Lipid Res., 1, 79(1960).

53. Radin, N. S., and Y. Akahori, J. Lipid Res.., 2. 335(1961).

54. Kenk, E. and W. Geilin, Hoppe-Seyler's Z. physiol. Chem., 326, 144(1961).

55. Cumins, J. T., and H. McIlwain, Blochem. J., 76, 64P(1960).

56. MeIlwain, H., B1ochem. J., 23, 514(1959).

57. MeIlwain, H., Biochem. J., 28, 24(1961).

58. Wolfe, L. S., and H. MeIlwain, Blochem. J., 28, 33(1961).

59. Wolfe, I. S., Biochem. J., 79, 348(1961).

60. Booth, D. A., J. Neurochem., 2, 265(1962).

61. Wolfo, L. S., Blochem. J., Z7, $9 P(1960)$.

62. Jâmofeldt, J., Bioch1m. ot Blophys. Acta, 48, 104, 111(1961). 
63. Deul.. D. H., and H. McIwain, J. Neurochem., 8, 256(1962). 64. Hillman, H., J. Neurochem., 8, 275(1961).

65. Thomson, C. G., and H. MeInwain, Blochem, J., 72, 342(1961).

66. Bogoch, S., V1rology, 4. 458(1957).

67. van Heyningen, W. E., and P. A. Miller, J. Gen. Microbiol., 24. $107(1961)$.

68. North, E. A., and H. M. Doery, Brit. J. Exptl. Pathol., 42, 23(1961).

69. van Heyningen, W. E., Brit. J. Exptl. Pathol., 42, 397(1961).

70. Bernheimer, $\Lambda_{0} W_{0}$, and $W_{0}$ E. van Heyninger, J. Gen. Microbiol., 24. $221(1961)$.

71. Jamieson, G. A., and M. D. Poul1k, Fed. Proc., 21, 230(1962).

72. Burton, R. M., and V. McB. Belfour, Blochem. Pharmacol., 2, 436(1962).

73. Irwin, R. L., and E. G. Trams, J. Pharmacol. Therapeutics, 137. $242(1962)$.

74. Johnson, G. A., and R. B. McCluer, Proc. Soc. Bxptl. Biol. Med.. 107. $692(1961)$.

75. Svennerholm, I., Acta Soc. Med. Upsaliensis, 62, 1(1957).

76. Long, C., and D. A. Staples, Biochem. J., 73, 385(1959).

77. Svennerholm, I., Biochim. et Biophys. Acta, 24, 604(1957).

78. Mlettinen, T., and I. T. Takkl Luukkainen, Acta Chem. Scand., 13. 856(1959).

79. Warren, I., J. B101. Chem.. 234, 1971(1959).

80. Edgar, G. F. W., and G. Smits, J. Neuropathol. Expt1. Neurol.. 17. $296(1958)$.

81. MeCluer, R. H., E. H. Coram, and H. S. Lee, J. Lipid Res., 3. 269(1962).

82. Trams, E. G., L. E. Guiffrida, and A. Karnen, Nature, 193. $680(1962)$

83. Kenk, E.. and W. Geilin, Hoppe-Seyler's Z, phys101, Chem., 326. 158(1961). 
84. Prostenik, M., and B. Majhofer-Orescanin, Naturwiss., 47, 399(1962).

85. Stanecev, N. Z., and E. Rosenberg, Blochim. et Blophys. Acta, 52. $733(1962)$.

86. Klenk, E., and K. Lauenstein, Hoppo-Seyler's Z. physiol. Chem.. 295. 164(1953).

87. Meltzer, H., J. Biol. Chem., 233, 1327(1958).

88. Kuhn, R., in Garbohydrate Gheinistry of Substances of Biological Interest. Vol. 1, M. L. Wolfrom, od.. (IVth Internationel Congress Biochem., Vienna (1958)), Pergamon Press, Inc., 1959. Symposium I. P. 67.

89. Klenk, E., and W. Geilin, Hoppe-Seyler's Z, physiol. Chem., 323. 126(1961).

90. Svennerholm, I., and A. Razl, Biochim. et Biophys. Acta, 53. 422(1961).

91. Dain, J. A., H. Weicker, G. Schmidt, and S. J. Thannhauser, in Cerebre1 Sphingolipldoses: 'A Srmposium on Tey-Sachs' Disesse and Al1ied Disorders, Academic Press, 1962, p. 289.

92. Stahl, E., Chem. Ztg., 82, 323(1958).

93. Wagner, H., L. Hörhammer, and P. Wolff, Biochem. A., 334 , $175(1961)$.

94. Johnson, G. A., K. Sambasivarao, and R. H. MeCluer, Fod. Proc., 21. $283(1962)$.

95. Svennerholm, L., Blochem. Biophys. Res. Communs., 2, 436(1962).

96. Wolfe, L. S., Canadian J. Biochem. Physiol., 40, 1261(1962).

97. Rosenberg, A., and E. Chargaff, Biochim. et Biophys. Acta, 42. 357 (1960).

98. Karkas, J. D., and E. Chargaff, Bioch1m. et Biophys. Acta, 42. $359(1960)$.

99. Martensson, E., A. Raal, and L. Svennerholm, Biochim. et Biophys. Acta, 30, 124(1958).

100. Svennerholm, I., Acte Soc. Med. Upsaliensis, 61, 287(1956).

101. Morris, D. L., Sclence, 107, 254(1948). 
102. Hodge. J. E.. and B. T. Hofreiter, in Methods in Carbohrdrate Chemlistry, Vol. 1, R. I. Whistler and M. I. Wolfrom eds.. Acadentc Press, 1962, p. 388.

103. Salfer, A., and S. Gerstenfeld, J. Lab. Clin. Med., 51, $448(1958)$. 104. Lauter, C. J., and E. G. Trams, J. Iipid Res., 3, 136(1962).

105. Harris, W. D., and P. Popat, J. Am. Oil Chemists' Soc., 21. $124(1954)$.

106. Block, R. J., E. I. Durrum, and G. Zweig, A Manual of Paper Chromatographv and Paper Electrophoresis, Academic Press, 1955.

107. Mangold, H., J. Am. Oil Chemists' Soc.. 38, 708(1961).

108. Hernandez, R., R. Hernandez, Jr., and I. R."Axelrod, Anal. Chem., 33. $370(1961)$.

109. Svennerholw, I., and E. Svennerholm, Nature, 181, 1154(1958).

110. Cardini. D. E., and L. F. Leloir, J. Biol. Chem., 225, 325(1957).

111. Warner, H. R., and W. E. M. Lands, J. Lip1d Res.. 1, 248(1960).

112. Svennerholm, I., personal communication.

113. Makita, A., and T. Yamakawa, J. Biochem. (Tokyo), 51, 124(1962).

114. Devor, A. W., personal communication.

115. Mïidner, H. G., J. R. Wherrett, and J. N. Cumings, J. Neurochem., 2. $607(1962)$.

116. Yokoyama, M., and E. G. Trams, Fed. Proc., 21, 25(1962).

117. Hough, I., and J. K. N. Jones, in Methods in Carbohidrate Chemistry, Vol. 1, R. L. Whistler and M. L. Wolfrom ods.. Academic Press, 1962, p. 24.

118. Trevelyan, W. E.: D. P. Procter, and J. S. Harrison, Nature. 166. $444(1950)$. Cited by Hough, L., and J. K. N. Jones. in Yethods in Carbohrdrate Chemistry. Vol. 1, p. 28. 


\section{AUTOBIOGRAPHY}

I, Garland Alton Johnson, was born in Laona, Wisconsin, July 16, 1936. I attended the public schools of Ioona and was graduated from Laona High School in May, 1954. I attended Carroll College, Waukesha, Wisconsin, and was graduated with a B. S. degree in June, 1958. I entered The Ohio State University in September, 1958 as a graduate teaching assistant in the Department of Physiological Chemistry and Pharmacology. The M. Sc. degree was granted me in June, 1960. While completing the requirements for the Doctor of Philosophy degree, I have been a graduate teaching assistant and have held a predoctoral fellowship from the National Institutes of Health. 